\title{
LATTICES OF HOMOMORPHISMS
}

\author{
B. A. DAVEY and H. A. PRIESTLEY \\ (Received 2 April 1984; revised 29 December 1984) \\ Communicated by R. Lidl
}

\begin{abstract}
Given a variety $\mathbf{K}$ of lattice-ordered algebras, $A \in \mathbf{K}$ is catalytic if for all $B \in \mathbf{K}, \mathbf{K}(A, B)$ is a lattice for the pointwise order. The catalytic objects are determined for various varieties of distributivelattice-ordered algebras. The characterisations obtained do not show an overall unity and exhibit diverse behaviour. Duality is employed extensively. Its usefulness in this context depends on the existence of an order-isomorphism between $\mathbf{K}(A, B)$ and the corresponding dual hom-set. Criteria for the existence of such an order-isomorphism are investigated for dualities of the Davey-Werner type. The relationship between catalytic objects and colattices is also discussed.
\end{abstract}

1980 Mathematics subject classification (Amer. Math. Soc.): 03 G 10, 06 A 10, 06 D 15, 18 D 35, 54 F 05.

\section{Introduction}

Kucera and Sands (1978) considered the following problem: given a variety $\mathbf{K}$ of lattices, under what conditions on $A \in \mathbf{K}$ is it true that, for all $B \in \mathbf{K}$, the set $\mathbf{K}(A, B)$ of homomorphisms from $A$ to $B$ is a lattice under the pointwise ordering? We shall be concerned with the structure of hom-sets in categories of distributive-lattice-ordered algebras and in dually equivalent categories.

Suppose that $\mathrm{C}$ is a category. For $A, B \in \mathrm{C}$, denote by $\mathrm{C}(A, B)$ the set of $C$-morphisms from $A$ to $B$. There are two natural hom-functors from $C$ to Set:

$$
\mathrm{C}(A,-): B \mapsto \mathrm{C}(A, B) \text { and } \mathrm{C}(-, B): A \mapsto \mathrm{C}(A, B) \text {. }
$$

This research was carried out while the second author held a visiting research fellowship at La Trobe University and was supported by La Trobe University and ARGS grants B8115287I and B8315711I. C) 1986 Australian Mathematical Society 0263-6115/86 \$A2.00+0.00 
For many of the categories $\mathbf{C}$ we wish to consider, $\mathbf{C}(A, B)$ has, defined in a natural way, more structure than merely that of a set. Suppose $\mathbf{X}$ is a concrete category, so that we have an underlying set functor $|-|: \mathbf{X} \rightarrow$ Set. We may then ask whether either of the natural hom-functors factors through $\mathbf{X}$.

Suppose now that $\mathbf{C}$ is a category of sets with structure and that the objects in $\mathbf{C}$ are equipped with a natural order which is preserved by $\mathbf{C}$-morphisms. (This is the case whenever $\mathbf{C}$ is a variety of lattice-ordered algebras.) These assumptions imply that, for any $A, B \in \mathbf{C}, \mathbf{C}(A,-)$ and $\mathbf{C}(-, B)$ can be factored through Poset (the category of ordered sets). For every $C \in \mathbf{C}$, the hom-sets $\mathbf{C}(A, C)$ and $\mathrm{C}(C, B)$ are then pointwise ordered. We shall say that $A \in \mathrm{C}$ is lattice-catalytic (in $C$ ) if $\mathbf{C}(A, B)$ is, for every $B \in \mathbf{C}$, a lattice in the pointwise order; $B$ is said to be lattice-cocatalytic (in $\mathbf{C}$ ) if $\mathbf{C}(C, B)$ is, for every $C \in \mathbf{C}$, a lattice in the pointwise order. We shall customarily drop the prefix "lattice", since the only other catalyticity notion we introduce (semilattice-catalytic, defined in the obvious way) we need only briefly. The set of catalytic objects in $\mathbf{C}$ is denoted by $\operatorname{Cat}(\mathbf{C})$, that of cocatalytic objects in $\mathbf{C}$ by $\operatorname{Cocat}(\mathbf{C})$.

If $\mathrm{C}(A,-)$ factors through Lat (the category of all lattices), then $A$ is said to be a colattice in $\mathrm{C}$, while if $\mathrm{C}(-, B)$ factors through Lat, then $B$ is said to be a lattice in $\mathbf{C}$.

Let $\mathbf{D}$ be the variety of bounded distributive lattices. An algebraic characterisation of Cat(D) was given by Balbes (1980). Balbes' characterisation was transferred to the dual category $\mathbf{P}$ of compact totally order-disconnected spaces by Priestley (1982a); she showed that Cat(D) constitutes those objects whose dual spaces are lattices in $\mathbf{P}$. Wherever, as for $\mathbf{D}$ and $\mathbf{P}$, we have a full duality between categories $\mathbf{C}$ and $\mathfrak{X}$ established by contravariant adjoint functors $D: \mathbf{C} \rightarrow \mathfrak{X}$ and $E: \mathfrak{X} \rightarrow \mathrm{C}$, there is a natural bijection, given by $D$, between the hom-sets $\mathrm{C}(A, B)$ and $\mathfrak{X}(D(B), D(A))$. If the hom-sets carry a naturally induced pointwise order, and if the bijection above is actually an order-isomorphism, then $D(\operatorname{Cat}(\mathbf{C}))=$ Cocat $(\mathfrak{X})$ and $D(\operatorname{Cocat}(\mathbf{C}))=\operatorname{Cat}(\mathfrak{X})$. This occurs for the D-P duality and also for its restriction to subcategories (not necessarily full). In Section 3 we exploit this fact to find $\mathrm{Cat}(\mathbf{C})$ for many varieties of distributive-lattice-ordered algebras. In many cases $|\operatorname{Cat}(\mathbf{C})|=|\mathbf{C}| \cap \operatorname{Cat}(\mathbf{D})$, where $|-|: \mathbf{C} \rightarrow \mathbf{D}$ is the forgetful functor. Interestingly it is possible to find examples in which $|\mathrm{Cat}(\mathbf{C})|$ is strictly larger, or strictly smaller, than $|\mathbf{C}| \cap \mathrm{Cat}(\mathbf{D})$.

In Section 4 we consider whether, in general, given a full duality of the type investigted by Davey and Werner (1983a) in which order structure is present in both categories, each hom-set is order-isomorphic to the corresponding dual hom-set. Section 5 is devoted to illustrative examples.

Finally, in Section 6 we interpret our earlier catalyticity characterisations in terms of lattices in dual categories. We discover that for many familiar varieties the catalytic objects are in fact colattices. In general we should not anticipate this, 
given the distinction between the factorisation of a hom-functor $\mathbf{C}(L,-): \mathbf{C} \rightarrow$ Set through Lat and through Poset; in the former case the map from $\mathrm{C}(L, A)$ to $\mathrm{C}(L, B)$ induced by a homomorphism $\phi \in \mathrm{C}(A, B)$ will be a lattice homomorphism, while in the latter case it is only known to be order-preserving.

\section{The D-P duality}

We shall make extensive use of the duality between the category $\mathbf{D}$ of bounded distributive lattices and the category $\mathbf{P}$ of compact totally order-disconnected spaces (Priestley spaces). We summarise below only the basic facts about the duality; further information can be found in the survey articles by Davey and Duffus (1982) and Priestley (1982c) and the references therein. For lattice theory we take Balbes and Dwinger (1974) as our reference; this contains full discussions of the algebraic properties of almost all the varieties we shall consider. The category theory we require can be found in Balbes and Dwinger (1974) and in Davey and Werner (1983a). Our notation is standard, except perhaps for our use of the symbols $\uparrow$ and $\downarrow$ : if $X$ is an ordered set and $Y \subseteq X$, then

$$
\begin{aligned}
& \uparrow Y=\{x \in X \mid x \geqslant y \text { for some } y \in Y\} \text { and } \\
& \downarrow Y=\{x \in X \mid x \leqslant y \text { for some } y \in Y\} .
\end{aligned}
$$

Given $y \in X$, we write $\uparrow y$ in place of $\uparrow\{y\}$ and $\downarrow y$ in place of $\downarrow\{y\}$.

By definition, an ordered topological space $(X ; \tau, \leqslant)$ belongs to $\mathbf{P}$ if $\tau$ is compact and, given $x \nless y$ in $X$, there exists a $\tau$-clopen set $U$ with $U=\uparrow U$ such that $x \in U$ and $y \notin U$.

The 2-element chain acts as a schizophrenic object living in both $\mathbf{D}$ and $\mathbf{P} ; \underline{2}$ denotes the chain in $\mathbf{D}$ with bottom element 0 and top element 1 , and 2 denotes the member of $\mathbf{P}$ obtained by giving the ordered set $\{0,1\}$, with $0<1$, the discrete topology. In categorical terms the duality may be stated as follows:

TheORem 2.1. The categories $\mathbf{D}$ and $\mathbf{P}$ are dually equivalent under the contravariant functors

$$
D:=\mathbf{D}(-, \underline{2}): \mathbf{D} \rightarrow \mathbf{P}
$$

(where the image $\mathbf{D}(A, 2)$ of $A \in \mathbf{D}$ has the pointwise order and inherits its topology from ${\underset{\sim}{2}}^{A}$ ) and

$$
E:=\mathbf{P}(-, 2): \mathbf{P} \rightarrow \mathbf{D}
$$

(where the image $\mathbf{P}(X, 2)$ of $X \in \mathbf{P}$ has pointwise operations). The unit $e: 1_{\mathbf{D}} \rightarrow$ $\mathbf{P}(\mathbf{D}(-, 2), 2)$ and counit $\varepsilon: 1_{\mathbf{P}} \rightarrow \mathbf{D}(\mathbf{P}(-, 2), 2)$ of the adjunction are given (for $A \in \mathbf{D}, X \in \mathbf{P})$ by $e_{A}, \varepsilon_{X}$, where

$$
e_{A}(a)(f)=f(a) \quad(a \in A, f \in \mathbf{D}(A, \underline{2}))
$$


and

$$
\varepsilon_{X}(x)(\phi)=\phi(x) \quad(x \in X, \phi \in \mathbf{P}(X, 2)) .
$$

These evaluation maps are isomorphisms.

On the object level, the duality allows us to identify $A \in \mathbf{D}$ with the lattice of clopen increasing subsets of its dual space $X=\mathbf{D}(A, \underline{2})$. The set $\mathbf{D}(A, \underline{2})$ is order-isomorphic to the set of prime filters of $A$ ordered by inclusion, and dually order-isomorphic to the set of prime ideals of $A$, also ordered by inclusion. In the survey articles cited above, $A$ is identified with the lattice of clopen decreasing subsets of its ordered set of prime ideals, appropriately topologised. Since it is more natural, in the context of hom-sets, to work with 2-valued homomorphisms rather than with prime ideals, we have to reverse the order when quoting results from the survey articles.

The adjunction between $\mathbf{D}$ and $\mathbf{P}$ produces a natural bijection between $\mathbf{D}(A, B)$ and $\mathbf{P}(D(B), D(A))$ (for every $A, B \in \mathbf{D}$ ). We shall show that the bijection is always an order-isomorphism for the pointwise orders. A much more general result is proved in Section 4.

Lemma 2.2. Let $A, B \in \mathbf{D}$. Then the pointwise-ordered hom-sets $\mathbf{D}(A, B)$ and $\mathbf{P}(D(B), D(A))$ are isomorphic via the natural bijection $D$.

Proof. Let $h, k \in \mathbf{D}(A, B)$. Then

$$
\begin{aligned}
h & \leqslant k \text { in } \mathbf{D}(A, B) \Leftrightarrow(\forall a \in A) h(a) \leqslant k(a) \text { in } B \\
& \Leftrightarrow(\forall a \in A)(\forall \psi \in \mathbf{D}(B, \underline{2})) \psi(h(a)) \leqslant \psi(k(a)) \text { in } \underline{2} \\
& \Leftrightarrow(\forall \psi \in \mathbf{D}(B, \underline{2}))(\forall a \in A)(D(h)(\psi))(a) \leqslant(D(k)(\psi))(a) \text { in } \underline{2} \\
& \Leftrightarrow(\forall \psi \in \mathbf{D}(B, \underline{2})) D(h)(\psi) \leqslant D(k)(\psi) \\
& \Leftrightarrow D(h) \leqslant D(k) .
\end{aligned}
$$

Corollary 2.3. $\operatorname{Cat}(\mathbf{D})=E(\operatorname{Cocat}(\mathbf{P}))$.

We note that $\mathbf{D}$ contains the 1-element lattice 1 whose dual space is the empty set. Clearly $|\mathbf{D}(\underline{1}, B)| \leqslant 1$ for every $B \in \mathbf{D}$. We adopt the convention that the empty set is a lattice. Thus $\underline{1}$ is automatically in Cat(D).

We can now analyse in greater depth the results on Cat(D) obtained in Balbes (1980) and Priestley (1982a). We first characterise Cocat(P), working in $\mathbf{P}$ throughout. The proof of the following lemma is straightforward. 
Lemma 2.4. Let $X \in \mathbf{P}$ be a lattice. Then $X$ is a topological lattice if every clopen increasing set in $X$ is a union of clopen sets of the form $\uparrow k$ where $k \in X$ and similarly for decreasing sets.

Proposition 2.5. Suppose that $X \in \operatorname{Cocat}(\mathbf{P})$. Then $X$ is a topological lattice.

Proof. By hypothesis, $\mathbf{P}(Y, X)$ is a lattice for the pointwise order, for every $Y \in \mathrm{P}$. Taking $Y$ to be a 1-point space we see that $X$ itself is a lattice. We now aim to show that $X$ satisfies the topological conditions of Lemma 2.4.

We fix a point $a \in X$ and make the following choice for $Y$. We let $Y$ be the disjoint union of $X$ with an infinite antichain $S$ and topologize $Y$ by taking as a base for the open sets

$$
\begin{aligned}
& \{U \subseteq X \mid a \notin U, U \text { clopen in } X\}, \\
\text { and } \quad & \{T \subseteq S \mid T \text { is finite }\}, \\
& \{U \cup T \mid a \in U, U \text { clopen in } X, T \text { cofinite in } S\} .
\end{aligned}
$$

It is easily seen that $Y \in \mathbf{P}$. Informally, $Y$ is $X$ with an unordered 'spike' $S$ glued to it at $a$ in a topologically appropriate fashion. Define $\phi, \psi \in \mathbf{P}(Y, X)$ by $\phi(y)=a$ for all $y \in Y$, and

$$
\psi(y)= \begin{cases}y & \text { if } y \in X \\ a & \text { if } y \in S\end{cases}
$$

By hypothesis $\phi$ and $\psi$ have an infimum $\phi \wedge \psi$ in $\mathbf{P}(Y, X)$. Since the order on $\mathbf{P}(Y, X)$ is defined pointwise,

$$
(\phi \wedge \psi)(y) \leqslant \phi(y) \wedge \psi(y) \text { for all } y \in Y .
$$

In particular $(\phi \wedge \psi)(y) \leqslant a$ for all $y \in Y$. Suppose, by way of contradiction, that there exists $t \in S$ such that $(\phi \wedge \psi)(t)<a$. Define $\eta \in \mathbf{P}(Y, X)$ by

$$
\eta(y)= \begin{cases}(\phi \wedge \psi)(y) & \text { if } y \neq t \\ a & \text { if } y=t .\end{cases}
$$

Then $\phi \wedge \psi<\eta \leqslant \phi, \psi$, which is impossible. Hence $(\phi \wedge \psi)(s)=a$ for every $s$ in $S$. From the definition of the topology on $Y$, and from the continuity of $\phi \wedge \psi$, we obtain $(\phi \wedge \psi)(a)=a$.

Now take any clopen increasing set $W$ in $X$ and let $a$ be a minimal point of $W$ (which always exists). Construct $Y, \phi$ and $\psi$ as above and let $\zeta=(\phi \wedge \psi) \uparrow_{X}$. Note that $\zeta(a)=a$. The set $\zeta^{-1}(W)$ is increasing and contains $a$, so $\zeta^{-1}(W) \supseteq \uparrow a$. For every $x \in \zeta^{-1}(W), x \wedge a \geqslant \zeta(x) \in W$, whence $x \wedge a \in W$. Minimality of a implies that $x \in \uparrow a$. Hence $\uparrow a=\zeta^{-1}(W)$, and this is clopen increasing. Thus

$$
W=\bigcup\{\uparrow a \mid a \text { is minimal in } W\}
$$

is a union of sets of the required form. 
The same argument, based on the fact that $\mathbf{P}(Y, X)$ is a join-semilattice, shows that every clopen decreasing set in $X$ is a union of clopen decreasing sets $\downarrow k$.

THEOREM 2.6. Let $A \in \mathbf{D}$. Then the following are equivalent:

(i) $A \in \mathrm{Cat}(\mathrm{D})$;

(ii) $D(A) \in \operatorname{Cocat}(\mathbf{P})$;

(iii) $D(A)$ is a topological lattice;

(iv) for all $Y \in \mathbf{P}, \mathbf{P}(Y, D(A))$ is a lattice, the lattice operations being given pointwise.

Proof. The equivalence of (i) and (ii) is Corollary 2.3. Proposition 2.5 gives (ii) $\Rightarrow$ (iii). It is trivial that (iv) $\Rightarrow$ (ii) and it is easy to verify that (iii) $\Rightarrow$ (iv).

(iv) $\Rightarrow$ (ii). Assume that (iv) holds. Note that $D(A)$ is certainly a lattice, since we can take $Y$ to be a 1-element space. Since products in $\mathbf{P}$ are cartesian products, the coordinate projections $\pi_{1}$ and $\pi_{2}$ from $D(A) \times D(A)$ to $D(A)$ belong to $\mathbf{P}(D(A) \times D(A), D(A))$. In this lattice, $\vee$ and $\wedge$ are defined pointwise. Hence $\pi_{1} \vee \pi_{2}:(x, y) \mapsto x \vee y$ and $\pi_{1} \wedge \pi_{2}:(x, y) \mapsto x \wedge y$, for all $x, y \in D(A)$. Thus $D(A)$ is a topological lattice, since $\pi_{1} \wedge \pi_{2}$ and $\pi_{1} \wedge \pi_{2}$ are continuous.

The equivalence of (i) and (iii) was established by Priestley (1982a), using the algebraic characterisation of Cat(D) obtained by R. Balbes (1980). The key portion of the proof of the theorem is contained in Proposition 2.5. To show $X \in \mathbf{P}$ belongs to $\operatorname{Cocat}(\mathbf{P})$ it is sufficient to know that $\mathbf{P}(Y, X)$ is a lattice (i) for $Y$ such that $|Y|=1$, and (ii) for the family of spaces $Y$ obtained by gluing a spike to $X$ at each point. The proof of Proposition 2.5 is not a direct dualisation of Balbes' arguments for Cat(D), but Balbes did introduce the lattices when duals are the 'spike spaces'.

In the proofs of Theorem 2.6 and the results leading up to it, meets and joins were handled quite separately. By restricting attention to meets we can characterise the meet-semilattice-catalytic objects in $\mathbf{D}$.

Theorem 2.7. $A \in \mathbf{D}$ is meet-semilattice-catalytic in $\mathbf{D}$ if and only if $D\left(A_{0}\right)$ is an algebraic lattice equipped with the Lawson topology (where $A_{0}$ denotes the lattice obtained by adjoining a new zero element to $A$ ).

Proof. The space $D\left(A_{0}\right)$ is obtained by adjoining a topologically isolated maximum point to $D(A)$. Thus $D\left(A_{0}\right)$ is a down-complete meet-semilattice with unit, and hence a complete lattice. (For references on the order-theoretic properties of P-objects, see Priestley (1982c).) The result now follows directly from the Fundamental Theorem for Compact Totally Disconnected Semilattices (see VI.3.13 in Gierz et al. (1980)). 
Algebraic properties of lattices whose duals are algebraic lattices are discussed in Priestley (1982b).

\section{Catalyticity in equational subcategories of $D$}

Suppose we wish to find the catalytic objects in some equational subcategory $\mathbf{C}$ of $\mathbf{D}$ and suppose that it is possible to characterise the (not necessarily full) subcategory $\mathbf{Q}=D(\mathbf{C})$ of $\mathbf{P}$. Restriction of Lemma 2.2 to $\mathbf{C}$ and $\mathbf{Q}$ gives an order-isomorphism between $\mathbf{C}(A, B)$ and $\mathbf{Q}(D(B), D(A))$ for any $A, B \in \mathbf{C}$. Consequently $\operatorname{Cat}(\mathbf{C})=E(\operatorname{Cocat}(\mathbf{Q}))$. It is possible to find $\operatorname{Cocat}(\mathbf{Q})$ in particular cases by modifying the arguments used to prove Theorem 2.6.

However a more economical strategy is frequently available. Assume that $X \in \operatorname{Cocat}(\mathbf{Q})$, so that $\mathbf{Q}(Y, X)$ is a lattice for each $Y \in \mathbf{Q}$. A judicious choice (or several choices) for $Y$ will provide information about $X$ and thereby restrict the class of candidates for objects in $\operatorname{Cocat}(\mathbf{Q})$. This procedure may restrict the cocatalytic spaces so far that the surviving candidates may be directly checked for cocatalyticity. Alternatively we may be able to invoke the following lemma.

Lemma 3.1. Suppose that $\mathbf{Q}$ is a subcategory of $P$ and that $\hat{\mathbf{Q}}$ is a full subcategory of $\mathbf{Q}$ such that

(i) $\hat{\mathbf{Q}} \supseteq$ Cocat $(\mathbf{Q})$; and

(ii) there exists a function $F$ associating to

each non-empty $Z \in \mathbf{P}$ an object $F(Z) \in \hat{\mathbf{Q}}$

such that for all $X \in \hat{\mathbf{Q}}$

$$
\mathbf{P}(Z,|X|) \simeq \mathbf{Q}(F(Z), X),
$$

where $\simeq$ denotes isomorphism of pointwise-

ordered sets, and where $|-|: \hat{\mathbf{Q}} \rightarrow \mathbf{P}$ is the

forgetful functor.

Then $X \in \operatorname{Cocat}(\mathbf{Q})$ implies that $|X| \in \operatorname{Cocat}(\mathbf{P})$.

Proof. The hypotheses imply that, whenever $X \in \operatorname{Cocat}(\mathbf{Q}), \mathbf{P}(Z,|X|)$ is a lattice for all $Z \in \mathbf{P}$ and hence that $|X| \in \operatorname{Cocat}(\mathbf{P})$.

In the opposite direction we have the following lemma.

Lemma 3.2. Suppose that $\mathbf{Q}$ is a subcategory of $\mathbf{P}$ and that $X \in \mathbf{Q}$, regarded as an object in $\mathbf{P}$, is cocatalytic in $\mathbf{P}$.

(a) If, for every $Y \in \mathbf{Q}$ and $\phi, \psi \in \mathbf{Q}(Y, X)$, the pointwise infimum and supremum of $\phi$ and $\psi$ are $\mathbf{Q}$-morphisms, then $X \in \operatorname{Cocat}(\mathbf{Q})$. 
(b) Assume that $\mathbf{Q}$ is closed under finite products and that $X \in \mathbf{Q}$ is a lattice. Assume further that $X \times_{Q} X$ is a subspace of $X^{2}=X \times_{\mathrm{P}} X$, that the restrictions of the projection maps $\pi_{1}, \pi_{2}: X^{2} \rightarrow X$ are the canonical projections from $X \times_{\mathrm{Q}} X$ to $X$, and that $\vee, \wedge: X \times_{\mathbf{Q}} X \rightarrow X$ are $\mathbf{Q}$-morphisms. Then $X \in \operatorname{Cocat}(\mathbf{Q})$.

[Part (a) can be applied easily in most cases. Part (b) provides a sufficient condition for (a) to hold; customarily $X \times_{\mathrm{Q}} X=X^{2}$ and the assumptions in (b) reduce to the requirement that $\vee$ and $\wedge$ should be $Q$-morphisms.]

Proof. (a) is trivial. To prove (b) it is sufficient to show that, under the assumptions in (b), for any $Y \in \mathbf{Q}$, the pointwise supremum of $\phi, \psi \in \mathbf{D}(Y, X)$ is a $\mathbf{Q}$-morphism. (A similar argument then works for infimum.)

There exists $\eta \in \mathbf{Q}\left(Y, X \times_{Q} X\right)$ such that the following diagram commutes:

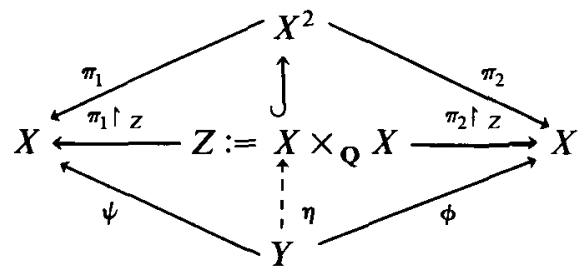

The map $y \mapsto(\phi \vee \psi)(y)=\phi(y) \vee \psi(y)$ is the composite of $\eta$ and the restriction of $\vee$ to $X \times_{\mathbf{Q}} X$, both of which are $\mathbf{Q}$-morphisms. Hence $\phi \vee \psi \in \mathbf{Q}(Y, X)$, as required.

The preceding lemmas suggest that we may be able to establish that for certain choices of $\mathbf{Q}$, Cocat $(\mathbf{Q})$ consists of those $\mathbf{Q}$-objects which are topological lattices in which $\vee$ and $\wedge$ are $\mathbf{Q}$-morphisms. Every topological lattice in $\mathbf{P}$ is the dual of a lattice which is both a double Stone algebra and a double Heyting algebra. Hence there are many subcategories $\mathbf{C}$ of $\mathbf{D}$ such that $\operatorname{Cat}(\mathbf{D}) \subseteq|\mathbf{C}|$ (where $1-1: \mathbf{C} \rightarrow \mathbf{D}$ is the forgetful functor), and in which we might reasonably expect to find non-trivial catalytic objects.

We now turn to examples. Since all our catalyticity characterisations follow the lines indicated above, it would be repetitious to give a full proof in each case. Accordingly we collect the key ingredients for the proofs in a table, located at the end of this section. We treat our first example - the variety of Stone algebras-in detail. The remaining proofs are obtained, mutatis mutandis, using the information in the table. Where new features emerge we draw attention to them.

We shall suppress forgetful functors except where it seems expedient to do otherwise. 
VARIETIES OF DISTRIBUTIVE $p$-ALGEBRAS. The non-trivial subvarieties of the variety $\mathbf{B}_{\omega}$ of distributive $p$-algebras form a chain

$$
\mathbf{B}_{0}(=\text { Boolean algebras }) \subset \mathbf{B}_{1}(=\text { Stone algebras }) \subset \mathbf{B}_{2} \subset \cdots \subset \mathbf{B}_{\omega} .
$$

The dual category $\mathbf{P}_{\omega}=D\left(\mathbf{B}_{\omega}\right)$ consists of those Priestley spaces $X$ for which $V$ clopen increasing in $X$ implies that $\downarrow V$ is clopen in $X$; the morphisms are those continuous order-preserving maps $\phi$ which map the maximal points above $x$ onto the maximal points above $\phi(x)$. For $1 \leqslant n<\omega, D\left(\mathbf{B}_{n}\right)$ is the full subcategory of $D\left(\mathbf{B}_{\omega}\right)$ consisting of those spaces in which each point is dominated by at most $n$ maximal points (see Davey and Duffus (1982) and Priestley (1982c); remember that the order has been reversed).

Theorem 3.3. For $1 \leqslant n \leqslant \omega, \operatorname{Cat}\left(\mathbf{B}_{n}\right)=\operatorname{Cat}(\mathbf{D})$, while $\operatorname{Cat}\left(\mathbf{B}_{0}\right)=\{\underline{1}, \underline{2}\}$.

Proof. Consider $n=1$. Suppose $\varnothing \neq X \in \operatorname{Cocat}\left(D\left(\mathbf{B}_{1}\right)\right)$. Let $Y$ be a 1element space, $\{u\}$, regarded as an element of $D\left(\mathbf{B}_{1}\right)$. Then

$$
\mathbf{P}_{\omega}(Y, X)=\left\{\phi_{x}: u \mapsto x \mid x \in \max X\right\},
$$

where $\max X$ denotes the set of maximal points of $X$. The maps $\phi_{x}$ and $\phi_{y}$ are comparable if and only if $x=y$. Since $\mathbf{P}_{\omega}(Y, X)$ is a lattice, $|\max X|=1$. Thus $X$ has a unique maximal point, 1 say.

We now apply Lemma 3.1. We take $\mathbf{Q}=D\left(\mathbf{B}_{1}\right)$ and

$$
\hat{\mathbf{Q}}=\{Y \in \mathbf{Q}|| \max Y \mid \leqslant 1\} .
$$

For $Z \in \mathbf{P}$, we define $F(Z)$ to be the space obtained from $Z$ by adjoining an isolated maximum point $1_{Z}$. For each $\phi \in \mathbf{P}(Z,|X|)$ we define $F(\phi): F(Z) \rightarrow X$ by requiring that $F(\phi) \uparrow_{Z}=\phi$ and $F(\phi)\left(1_{Z}\right)=1$. Clearly the map $\phi \mapsto F(\phi)$ sets up an order-isomorphism between $\mathbf{P}(Z,|X|)$ and $\mathbf{Q}(F(Z), X)$. It follows from Lemma 3.1 that $\operatorname{Cocat}\left(D\left(\mathbf{B}_{1}\right)\right) \subseteq\left\{X \in D\left(\mathbf{B}_{1}\right) \mid X \in \operatorname{Cocat}(\mathbf{P})\right\}$.

Now suppose that $\varnothing \neq X \in \operatorname{Cocat}(\mathbf{P})$. Since $X$ has a unique maximal, it follows that $X \in D\left(\mathbf{B}_{1}\right)$. On any space $Y \in D\left(\mathbf{B}_{1}\right)$ let $M$ denote the continuous map taking each point to the unique maximal point above it. For $Y \in D\left(\mathbf{B}_{1}\right)$ and $\phi, \psi \in \mathbf{P}_{\omega}(Y, X)$, we have $\phi \circ M=M \circ \phi$ and $\psi \circ M=M \circ \psi$. It follows that $\phi \wedge \psi$ and $\phi \vee \psi$ (defined pointwise) commute with $M$ and so are $\mathbf{P}_{\omega}$ morphisms. Hence $\mathrm{P}_{\omega}(Y, X)$ is a lattice under the pointwise operations, so that $X \in \operatorname{Cocat}(\mathbf{Q})$.

For $1<n \leqslant \omega$, the result is proved in the same way; see the table. That the one- and two-element algebras are the only catalytic Boolean algebras was proved in Balbes (1980). For completeness we note here that it is trivial that $X \in$ $\operatorname{Cocat}\left(D\left(\mathbf{B}_{0}\right)\right)$ implies $|X| \leqslant 1$; consider maps from a 1-point space into $X$. 
VARIETIES OF DISTRIBUTIVE DOUBLE $p$-ALgebras. The variety $\mathbf{B}_{\omega}^{\omega}$ has an uncountable lattice of subvarieties. We consider here only the subvarieties $\mathbf{B}_{n}^{m}$ $(1 \leqslant m, n \leqslant \omega) ; A \in \mathbf{B}_{n}^{m}$ if and only if $A \in \mathbf{B}_{n}$ and its order-theoretic dual $A^{d} \in \mathbf{B}_{m}$. The dual category $D\left(\mathbf{B}_{\omega}^{\omega}\right)$ consists of those spaces $X$ in $\mathbf{P}$ for which, for every clopen increasing subset $V$, the sets $\downarrow V$ and $\uparrow(X \backslash V)$ are clopen. Morphisms are those P-morphisms which map the maximals above $x$ onto the maximals above $\phi(x)$, and similarly for the minimals. The information in the table makes it straightforward to prove the following theorem.

Theorem 3.4. For $1 \leqslant m, n \leqslant \omega, \operatorname{Cat}\left(\mathbf{B}_{n}^{m}\right)=\operatorname{Cat}(\mathbf{D})$

Varieties of Heyting algebras. We denote by $\mathbf{H}$ the variety of Heyting algebras. The dual category $Z=\mathrm{D}(\mathbf{H})$ consists of those spaces $X \in \mathbf{P}$ such that $\downarrow U$ is open for any open set $U \subseteq X$. Let $X, Y \in \mathbf{Z}$. A map $\phi \in \mathbf{P}(X, Y)$ is a Z-morphisms if and only if $\phi(\uparrow x)=\uparrow \phi(x)$ for all $x \in X$.

We do not have a complete characterisation of $\operatorname{Cat}(\mathbf{H})$. Easier to handle are the bounded relative Stone algebras which form a subvariety $\mathbf{L}_{\omega}$ of $\mathbf{H}$ equationally defined by $A \in \mathbf{L}_{\omega}$ if and only if

$$
(\forall a, b \in A) \quad(a \rightarrow b) \vee(b \rightarrow a)=1 .
$$

Dually, $A \in \mathbf{L}_{\omega}$ if and only if, for every $x \in D(A), \uparrow x$ is a chain. The non-trivial subvarieties of $\mathbf{L}_{\omega}$ form a chain

$$
\mathbf{L}_{2} \subset \mathbf{L}_{3} \subset \cdots \subset \mathbf{L} \omega \text {. }
$$

For $2 \leqslant n<\omega, \mathbf{L}_{n}=\mathbb{I S P}(\underline{n})$ and $D\left(\mathbf{L}_{n}\right)$ consists of those spaces in $D\left(\mathbf{L}_{\omega}\right)$ for which $|\uparrow x| \leqslant n-1$ for every $x \in X$.

Lemma 3.5. (i) If $X \in \operatorname{Cocat}\left(D\left(\mathbf{L}_{\omega}\right)\right)$, then $X$ is a chain.

(ii) If $X \in \operatorname{Cocat}\left(D\left(\mathbf{L}_{n}\right)\right)(2 \leqslant n<\omega)$, then $X$ is a chain (with at most $n-1$ elements).

Proof. First note that, in both cases, either $X=\varnothing$ or $X$ has a unique maximal element, 1 say. (To prove this, consider $\mathbf{Z}$-morphisms from a one-element space into $X$.) Let $X \in \operatorname{Cocat}\left(D\left(\mathbf{L}_{\omega}\right)\right)$. Take $x, y \in X$ and form $Y=\uparrow x \times \uparrow y$ with the lexicographic order:

$$
\left\langle u_{1}, v_{1}\right\rangle \leqslant\left\langle u_{2}, v_{2}\right\rangle \Leftrightarrow u_{1}<u_{2} \text { or }\left(u_{1}=u_{2} \text { and } v_{1} \leqslant v_{2}\right) \text {. }
$$

Since $X \in \mathbf{L}_{\omega}$, both $\uparrow x$ and $\uparrow y$ are complete chains, and hence $Y$ is also a complete chain. Now endow $Y$ with the interval topology. This is compact due to the fact that $Y$ is complete. Since both $\uparrow x$ and $\uparrow y$ are algebraic, they both satisfy

$$
u<v \Rightarrow(\exists s, t) \quad u \leqslant s<t \leqslant v .
$$


It follows easily from this that $Y$ is totally order-disconnected. Hence $Y \in \mathbf{P}$, and since $Y$ is a chain, $Y \in D\left(\mathbf{L}_{\omega}\right)$ follows.

We define $\phi, \psi \in \mathbf{Z}(Y, X)$ by

$$
\phi(\langle u, v\rangle)=u \text { and } \psi(\langle u, v\rangle)=\left\{\begin{array}{lll}
v & \text { if } & u=x \\
1 & \text { if } & u>x
\end{array}\right.
$$

By construction, $\phi(\langle x, y\rangle)=x$ and $\psi(\langle x, y\rangle)=y$. By hypothesis, $\phi$ and $\psi$ have an infimum in $Z(Y, X)$ which at each point is dominated by both $\phi$ and $\psi$. It follows that $x$ and $y$ have a common lower bound, say $z$. Since $\uparrow z$ is a chain, $x$ and $y$ are comparable. Hence $X$ is a chain.

Let $X \in \operatorname{Cocat}\left(D\left(\mathbf{L}_{n}\right)\right)(2 \leqslant n<\omega)$, and by way of contradiction suppose that $X$ is not a chain. Since $X$ has a maximum element, and since chains in $X$ have fewer than $n$ elements, we can write $X$ ( qua ordered set) as $X_{1} \oplus(k-1)$ where $1 \leqslant k<n$, and where $X_{1}$ has at least two maximal points. Let $\underset{\sim}{k}$ be the $k$-element chain with the discrete topology. Then $\mathbf{Z}(\underset{\sim}{k}, X)$ has minimal elements $f_{x}: \underset{\sim}{k} \rightarrow X$ defined by $f_{x}(j)=j-1(1 \leqslant j \leqslant k-1)$, and $f_{x}(0)=x$, where $x$ ranges over $\max X_{1}$. For $x \neq y$ in $\max X_{1}, f_{x}$ and $f_{y}$ have no lower bound, and we have the required contradiction.

Lemma 3.6. Let $X$ be a chain in $D\left(\mathbf{L}_{n}\right)(2 \leqslant n \leqslant \omega)$. Then $X \in \operatorname{Cocat}\left(D\left(\mathbf{L}_{n}\right)\right)$.

Proof. Let $Y \in D\left(\mathbf{L}_{n}\right)$, and take $\phi, \psi \in \mathbf{Z}(Y, X)$. Since $X \in \operatorname{Cocat}(\mathbf{P}), \phi \wedge \psi$ and $\phi \vee \psi$, defined pointwise, are $\mathbf{P}$-morphisms.

To show that $\phi \wedge \psi$ is a Z-morphism, it is enough to show that, for every $x \in Y, \uparrow(\phi(x) \wedge \psi(x)) \subseteq(\phi \wedge \psi)(\uparrow x)$. (The reverse inclusion holds because $\phi \wedge \psi$ is order-preserving.) Assume without loss of generality that $\phi(x) \leqslant \psi(x)$ and let $v \in \uparrow(\phi(x) \wedge \psi(x))=\uparrow \phi(x)$. Since $\phi$ is a Z-morphism, there exists $y \in \uparrow x$ such that $\phi(y)=v$. If $\psi(y) \geqslant v$, then $(\phi \wedge \psi)(y)=v$, as we require. If $\psi(y)<v$, then there exists $z \in \uparrow y$ such that $\psi(z)=v$. In this case, $\phi(z) \geqslant$ $\phi(y)=v$, and so $(\phi \wedge \psi)(z)=\psi(z)=v$, as required.

Now let $v \in \uparrow(\phi(x) \vee \psi(x))$. Then, without loss of generality, $v \geqslant \psi(x) \geqslant$ $\phi(x)$. There exist $y, z \geqslant x$ such that $v=\psi(y)=\phi(z)$. Since $Y \in D\left(\mathbf{L}_{\omega}\right), y$ and $z$ are comparable. Suppose $y \leqslant z$. Then $\phi(y) \leqslant \phi(z)=\psi(y)$, and so $v=$ $(\phi \vee \psi)(y)$. Similarly, if $y \geqslant z$, then $\psi(z) \leqslant \psi(y)=\phi(z)$, and so $v=$ $(\phi \vee \psi)(z)$. It follows that $\phi \vee \psi$ is a Z-morphism.

From the last two lemmas we deduce immediately

TheOREM 3.7. (i) $\operatorname{Cat}\left(\mathbf{L}_{\omega}\right)=\left\{A \in \mathbf{L}_{\omega} \mid A\right.$ is a chain $\}=\mathbf{L}_{\omega} \cap \operatorname{Cat}(\mathbf{D})$.

(ii) For $2 \leqslant n<\omega, \operatorname{Cat}\left(\mathbf{L}_{n}\right)=\{\underline{1}, \underline{2}, \ldots, \underline{n}\}=\mathbf{L}_{n} \cap \operatorname{Cat}(\mathbf{D})$. 
For $\mathbf{H}$ the situation is more complicated. Joins in dual hom-sets may be defined, but not pointwise, as the following example shows. We take $Y$ to be the 4-element Boolean algebra $\underline{2}^{2}$ with elements $0, a, b$ and 1, and we take $X$ to be the 3-element chain with elements $0<c<1$. Define $\phi, \psi: Y \rightarrow X$ by

$$
\phi(0)=\psi(0)=0, \quad \phi(a)=\psi(b)=c, \quad \phi(b)=\psi(a)=\psi(1)=1 .
$$

These are $\mathbf{Z}$-morphisms whose pointwise supremum maps 0 to 0 and the remaining elements of $\underline{2}^{2}$ to 1 . The supremum of $\phi$ and $\psi$ in $\mathbf{Z}(Y, X)$ is, however, the map taking 0 to $c$ and the remaining elements to 1 .

\section{THEOREM 3.8. Every finite chain is catalytic in $\mathbf{H}$.}

Proof. We must show that any finite chain in $\mathbf{Z}$ is cocatalytic. The first part of the proof of Lemma 3.6 does not require that $X \in D\left(\mathbf{L}_{\omega}\right)$, but only that $X \in \mathbf{Z}$. Therefore any finite chain in $\mathbf{Z}$ is meet-semilattice-cocatalytic.

Let $X$ be the discretely topologised chain with elements $c_{0}<c_{1}<\cdots<c_{n-1}$ $(n \geqslant 1)$ and suppose that every finite chain of cardinality less than $n$ is join-semilattice-cocatalytic. Fix $Y \in \mathbf{Z}$ and $\phi, \psi \in \mathbf{Z}(Y, X)$. Define sets $Y_{0}, Y_{1}, \ldots$ and maps $\gamma_{0}, \gamma_{1}, \ldots$ as follows:

$$
\begin{gathered}
Y_{0}=\phi^{-1}\left(c_{0}\right) \cup \psi^{-1}\left(c_{0}\right), \\
\gamma_{0}(x)= \begin{cases}c_{0} & \text { if } x \in Y_{0}, \\
(\phi \vee \psi)(x) & \text { if } x \in Y \backslash Y_{0},\end{cases}
\end{gathered}
$$

(the join of $\phi \uparrow_{Y \backslash Y_{0}}$ and $\psi \uparrow_{Y \backslash Y_{0}}$ exists in $Z\left(Y \backslash Y_{0}, \uparrow c_{1}\right)$ by the inductive hypothesis); for $0 \leqslant k \leqslant n-2, Y_{k+1}=Y_{k} \backslash \downarrow \gamma_{k}^{-1}\left(c_{k+1}\right)$,

$$
\gamma_{k+1}(x)=\left\{\begin{array}{ll}
c_{k+1} & \text { if } x \in Y_{k+1} \\
\gamma_{k}(x) & \text { if } x \notin Y_{k+1}
\end{array} .\right.
$$

We claim that $\gamma:=\gamma_{n-1}$ is the required supremum of $\phi$ and $\psi$ in $\mathbf{Z}(Y, X)$. (In fact $\gamma_{n-1}=\gamma_{n-3}$, but we do not need to know this.) Trivially, we have $\gamma \geqslant \phi, \psi$. Suppose $\zeta \in \mathbf{Z}(Y, X)$ dominates $\phi$ and $\psi$. Consideration of $\zeta \Gamma_{Y \backslash Y_{0}}$ shows that $\zeta \geqslant \gamma_{0}$ on $Y \backslash Y_{0}$, whence $\zeta \geqslant \gamma_{0}$. Now assume that $\zeta \geqslant \gamma_{k}$ and suppose by way of contradiction that there exists $x \in Y_{k+1}$ such that $\zeta(x)<\gamma_{k+1}(x)=c_{k+1}$. Then $c_{k}=\gamma_{k}(x) \leqslant \zeta(x)<c_{k+1}$, so that $\gamma_{k}(x)=\zeta(x)$. Since $\zeta$ is a $\mathbf{Z}$-morphism, there exists $y \geqslant x$ such that $c_{k+1}=\gamma_{k}(y)$. This implies that $x \in \downarrow \gamma_{k}^{-1}\left(c_{k+1}\right)$, which is impossible if $x \in Y_{k+1}$. By induction we obtain $\zeta \geqslant \gamma$. It remains to prove that $\gamma \in \mathbf{Z}(Y, X)$. In $Y$, if $U$ is clopen, then $\downarrow U$ is clopen, so that (again by induction) $\gamma$ is continuous. Since each of the sets $\downarrow \gamma_{k}^{-1}\left(c_{k+1}\right)$ is decreasing, $\gamma$ is order-preserving. We shall now prove by induction that, for each $k, \gamma_{k} \uparrow_{Y \backslash Y_{k}} \in$ $\mathbf{Z}\left(Y \backslash Y_{k}, X\right)$. Since $Y_{n-1}=\varnothing$, it will follow that $\gamma=\gamma_{n-1} \in \mathbf{Z}(Y, X)$. Clearly $\gamma_{0} \uparrow_{Y \backslash Y_{0}}$ is a $\mathbf{Z}$-morphism. Suppose $\gamma_{k} \uparrow_{Y \backslash Y_{k}}$ is a $\mathbf{Z}$-morphism. Let $x \in Y \backslash Y_{k+1}$. 
Then $\gamma_{k+1}(x)=\gamma_{k}(x)$. Let $v \geqslant \gamma_{k+1}(x)$. If $x \in Y \backslash Y_{k}$, there exists $y \in Y \backslash Y_{k}$, $y \geqslant x$, with $\gamma_{k}(y)=v$. Then $v \in \gamma_{k+1}(\uparrow x)$, as we require. Now suppose that $x \in$ $Y_{k} \backslash Y_{k+1}$, so that $x \in \downarrow \gamma_{k}^{-1}\left(c_{k+1}\right)$. In this case there exists $y \geqslant x$ with $\gamma_{k}(y)=$ $c_{k+1}$. Necessarily $y \notin Y_{k}$ (by definition of $\gamma_{k}$ ), and so, because $\gamma_{k} \uparrow Y \backslash Y_{k}$ is a $\mathbf{Z}$-morphism, any $w \geqslant c_{k+1}$ is such that $w=\gamma_{k}(z)$ for some $z \geqslant y \geqslant x$. But $\gamma_{k+1}(x)=c_{k}$. Either $v \geqslant c_{k+1}$, in which case $v \in \gamma_{k+1}(\uparrow x)$ as we require, or $v=c_{k}$, in which case $v \in \gamma_{k+1}(\uparrow x)$ trivially. Hence $\gamma_{k+1} \uparrow_{Y \backslash Y_{k+1}}$ is a Z-morphism on $Y \backslash Y_{k+1}$.

If one merely requires an example of a space $X \in Z$ which is cocatalytic and such that joins in $\mathbf{Z}(Y, X)$ are not always defined pointwise, a simplified version of the argument above shows that $X$ can be taken to be a 3-element chain.

VARIETIES OF OCKhaM ALgebras. Let $\mathbf{C}$ be a subcategory of $\mathbf{D}$. In the examples we have discussed so far, it has turned out that $|\operatorname{Cat}(\mathbf{C})|=\operatorname{Cat}(\mathbf{D}) \cap|\mathbf{C}|$, where $|-|: \mathbf{C} \rightarrow \mathbf{D}$ is the forgetful functor. Varieties of Ockham algebras provide examples showing that $|\mathrm{Cat}(\mathbf{C})|$ may be strictly larger, or strictly smaller, than $\operatorname{Cat}(\mathbf{D}) \cap|\boldsymbol{C}|$.

A (distributive) Ockham algebra is a bounded distributive lattice $(A ; \wedge, \vee, 0,1)$ with a unary operation satisfying

$$
\sim(a \wedge b)=\sim a \vee \sim b, \quad \sim(a \vee b)=\sim a \wedge \sim b, \quad \sim 0=1, \quad \sim 1=0 .
$$

The class of all such algebras forms a variety $\mathbf{O}$, which has been extensively studied by M. S. Goldberg (1981), following initial investigations by A. Urquhart (1979). Under the D-P duality, $\mathbf{O}$ is dual to the category $\mathbf{S}$ of Ockham spaces: $\mathbf{S}:=\{(X ; g) \mid X \in \mathbf{P}$ and $g: X \rightarrow X$ is continuous and order-reversing $\} ; g(x)(a)$ $=1 \Leftrightarrow x(\sim a)=0$ (for $a \in A, x \in D(A)$ ). Any $X \in \mathbf{P}$ can be made into an Ockham space by selecting a point $u \in X$ and defining $g(x)=u$ for all $x \in X$; such a space corresponds to an Ockham algebra $A$ in which the negation $\sim$ is given by

$$
\sim a= \begin{cases}1 & \text { if } a \in I, \\ 0 & \text { if } a \notin I,\end{cases}
$$

for some prime ideal $I$ of $A$. We shall call such algebras prime.

The variety $\mathbf{O}$ is $\operatorname{ISP}\left(L_{\omega}\right)$, where $D\left(L_{\omega}\right)=\beta \mathbb{N}$ (the Stone-Čech compactification of the natural numbers with the discrete topology), and where $g$ is the continuous extension to $\beta \mathbb{N}$ of the successor map on $\mathbb{N}$. Important subvarieties of $\mathbf{O}$ are the varieties $\mathbf{P}_{m, n}(m>n \geqslant 0)$, where $\mathbf{P}_{m, n}$ is generated by the algebra $L_{m, n}$ whose dual is the Ockham space $m_{n}:=\left(\mathbb{Z}_{m} ; \gamma\right)$ in which $\gamma: \mathbb{Z}_{m} \rightarrow \mathbb{Z}_{m}$ satisfies $\gamma(k)=k+1(0 \leqslant k<m-1)$ and $\gamma(m-1)=n$. It is shown in Urquhart (1979) that the dual category $\mathbf{S}_{m, n}=D\left(\mathbf{P}_{m, n}\right)$ is given by

$$
\mathbf{S}_{m, n}=\left\{(X ; g) \in \mathbf{S} \mid g^{m}=g^{n}\right\} \text {. }
$$


We note that $\mathbf{P}_{2,0}$ is the variety of de Morgan algebras. Our first theorem follows the pattern of earlier results.

Theorem 3.9. (i) $\operatorname{Cat}(\mathbf{O})=\{L \in \mathbf{O} \mid L \in \operatorname{Cat}(\mathbf{D})$ and $L$ is a prime algebra $\}$;

(ii) For $m>n>0, \operatorname{Cat}\left(\mathbf{P}_{m, n}\right)=\left\{L \in \mathbf{P}_{m, n} \mid L \in \operatorname{Cat}(\mathbf{D})\right.$ and $L$ is a prime algebra ;

(iii) For $m>0$, $\operatorname{Cat}\left(P_{m, 0}\right)=\{\underline{1}, \underline{2}\}$.

Proof. Suppose $(X ; g) \in \operatorname{Cocat}(\mathbf{S})$. We consider the set $\mathbf{S}(\beta \mathbb{N}, X)$ of continuous functions $f_{x}: \beta \mathbb{N} \rightarrow X(x \in X)$, where $f_{x}(k)=g^{k}(x)$ for all $k \in \mathbb{N}$. We have

$$
\begin{gathered}
f_{x} \geqslant f_{y} \Leftrightarrow(\forall k \geqslant 0) \quad g^{k}(x) \geqslant g^{k}(y) \Leftrightarrow x \geqslant y \quad \text { and } \\
g(x)=g(y)
\end{gathered}
$$

(since $g$ is order-reversing). For any $x, y \in X, f_{x}$ and $f_{y}$ have a supremum, which is necessarily $f_{z}$ for some $z \in X$. But then $g(x)=g(z)=g(y)$, so $g$ is constant. Similarly, consideration of $\mathbf{S}\left(m_{n}, X\right)$, for $(X ; g) \in \operatorname{Cocat}\left(\mathbf{S}_{m, n}\right)$, shows that $g$ is constant in this case too. When $n=0$, the condition $g^{m}=1$ forces $|X| \leqslant 1$. The remainder of the proof is routine; see the table.

We now consider subvarieties of $\mathbf{O}$ which are generated by a single finite subdirectly irreducible algebra whose underlying lattice is not necessarily Boolean. It was proved by Urquhart (1979) that a finite algebra $\underline{P}$ is subdirectly irreducible if and only if it is a subalgebra of some $L_{m, n}$. The dual $(X ; g)$ of $\underline{P}$ is a space of the form $m_{n}$ equipped with an order relation with respect to which $g$ is order-reversing. The possible spaces of cardinality 1 or 2 are depicted below, the arrows indicating the action of $g$.

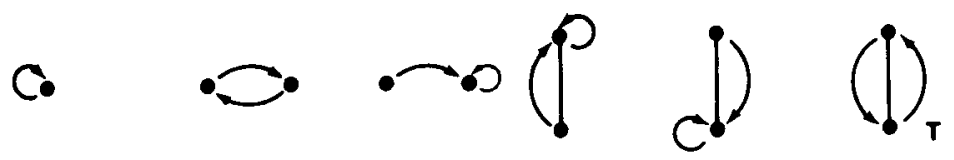

The associated varieties are Boolean algebras, de Morgan algebras $\left(\mathbf{P}_{2,0}\right), \mathbf{P}_{2,1}$, Stone algebras, dual Stone algebras, and Kleene algebras. All except the last are covered by our earlier discussions. The variety of Kleene algebras plays a significant role in later sections. It is generated by the 3-element chain $\{0, c, 1\}$, with $0<c=\sim c<1$. We denote this chain's dual Ockham space by $T$.

For the remainder of this section we take $\underline{P}$ to be a finite subdirectly irreducible algebra in $\mathbf{O}$ and $K:=\operatorname{MSP}(\underline{P})$ to be the variety it generates. We denote the dual space of $\underline{P}$ by $(X ; g)$, and we let $\mathfrak{X}=D(\mathbf{K})$. A semantic description of $\mathfrak{X}$ is given in Goldberg (1981), Theorem 2.11. In order to characterise Cocat $(\mathfrak{X})$ we need to refine this description. 
We adopt the notation of Goldberg (1981). We denote the collection of $g$-closed subsets of S-morphic images of $X$ by $G M(X)$; because $\mathrm{O}$ has the Congruence Extension Property, $G M(X)=M G(X)$. An element $e$ of $Y \in$ $G M(X)$ is called an end of $Y$ if $Y=\left\{g^{k}(e) \mid k \geqslant 0\right\}$, and $Y$ is said to be a loop if, for some $k>0, g^{k}=1$ on $Y$. A non-loop has a unique end, and every element of a loop is an end. For $Y \in G M(X)$ and $e$ an end of $Y$, define

$$
\sigma_{(Y, e)}(x):=\underset{0 \leqslant k, l \leqslant m}{\&}\left(g^{k}(x) \leqslant g^{l}(x) \mid g^{k}(e) \leqslant g^{l}(e) \text { in } Y\right),
$$

where $\&$ denotes conjunction. Let $\mathscr{P}$ be the set of all $(Y, e)$ such that

(i) $Y \in G M(X)$,

(ii) $e$ is an end of $Y$, with the proviso that, if $Y$ is a loop, then

(a) if $Y$ is an antichain, it occurs exactly once in $\mathscr{P}$ with an arbitrary but fixed point for $e$, and (b) if $Y$ is not an antichain, it occurs twice in $\mathscr{P}$, once with a minimal element as $e$, and once with a maximal element as $e$; moreover we assume that no two of the objects in $\mathscr{P}$ are isomorphic as pointed $\mathbf{S}$-objects.

Note that the characterisation of the possible orders on a loop $L$ (Urquhart (1979), Lemma 3, or Goldberg (1981), Lemma 2.16) implies that the height of $L$ is at most one. It follows that if $f$ is an end of $Z \in G M(X)$, then there exists $(Y, e) \in \mathscr{P}$ and an $\mathbf{S}$-isomorphism $\phi: Z \rightarrow Y$ with $\phi(f)=e$. Consequently, up to isomorphism of pointed $S$-objects, $\mathscr{P}$ allows for all $(Y, e)$ with $Y \in G M(X)$ and $e$ an end of $X$.

We order $\mathscr{P}$ by defining $(Y, e) \leqslant(Z, f)$ if and only if there is an $\mathbf{S}$-morphism $\phi: Z \rightarrow Y$ with $\phi(f)=e$. The following lemma is immediate.

LEMMA 3.10. $(Y, e) \leqslant(Z, f)$ in $\mathscr{P}$ if and only if, for all $X \in \mathbf{S}$, and for all $x \in X$,

$$
X \vDash \sigma_{(Y, e)}(x) \Rightarrow X \vDash \sigma_{(Z, f)}(x)
$$

Lemma 3.11. $(\mathscr{P}, \leqslant)$ has at most two maximals, $(X, e)$ and $\left(X_{1}, g(e)\right)$, where $X_{1}=g(X)$, and $e$ is a fixed end for $X$.

Proof. Since $g^{2}$ is an S-morphism, we have, for any $g$-subset $Y$ of $X$ and any end $f$ of $Y$, that $(Y, f) \leqslant(X, e)$ or $(Y, f) \leqslant\left(X_{1}, g(e)\right)$. Now let $Z \in G M(X)$ and let $j$ be an end of $Z$. Suppose $Z$ is a loop. Then $Z \in M(L)$, where $L$ is the loop of $X$ (that is, the subset of $X$ forming a loop). We can choose $f \in L$ with $(Z, j) \leqslant(L, f)$. Now assume that $Z$ is not a loop. In this case there exists $Y \in G(X)$ (with $Y$ not a loop) such that $(Z, j) \leqslant(Y, f)$, where $f$ is the unique end of $Y$. By transitivity we have $(Z, j) \leqslant(X, e)$ or $(Z, j) \leqslant\left(X_{1}, g(e)\right)$, as required. 
By considering Cases (0)-(4) preceding Corollary 2.15 in Goldberg (1981), it is easy to establish the following lemma.

LEMMA 3.12. $\left(X_{1}, g(e)\right)$ is maximal in $(\mathscr{P}, \leqslant)$ except when

(a) $|X| \leqslant 2$ and $X \neq T$,

(b) $X$ is an antichain,

(c) $X$ is isomorphic or dually isomorphic to either

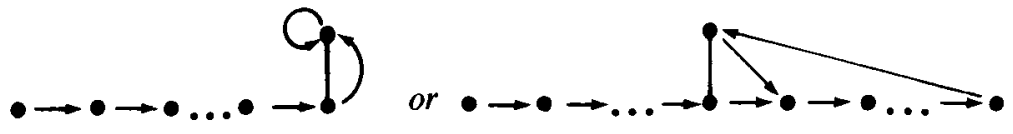

We can now describe $\mathfrak{X}$ in a convenient way. We let $W \in \mathfrak{X}$ and define, for $(Y, e) \in \mathscr{P}$,

$$
\begin{aligned}
W_{(Y, e)} & =\{x \in W \mid(\exists \phi \in S(Y, W)) \phi(e)=x\} \\
& =\left\{x \in W \mid W \vDash \sigma_{(Y, e)}(x)\right\} .
\end{aligned}
$$

Goldberg's description of $\mathfrak{X}$ (Goldberg (1981), Theorem 2.11) implies that

$$
W=\bigcup\left\{W_{(Y, e)} \mid(Y, e) \in \mathscr{P}\right\} .
$$

We order $\mathscr{P}_{W}:=\left\{W_{(Y, e)} \mid(Y, e) \in \mathscr{P}\right\}$ by set inclusion. The map $\alpha:(Y, e) \mapsto$ $W_{(Y, e)}$ is clearly order-preserving and onto. Since $\mathscr{P}$ has at most two maximals, it follows that $\mathscr{P}_{W}$ has at most two maximals.

THEOREM 3.13. Let $W \in \mathbf{S}$. Then the following conditions are equivalent:

(i) $W \in \mathfrak{X}$;

(ii) $W \vDash(\forall x)\left(\sigma_{(X, e)} \vee \sigma_{\left(X_{1}, g(e)\right)}\right)$;

(iii) $W$ is the (not necessarily disjoint) union of spaces

$$
W_{0}:=\{x \in W \mid(\exists \phi \in \mathbf{S}(X, W)) \phi(e)=x\}
$$

and

$$
W_{1}:=\left\{x \in W \mid\left(\exists \phi \in \mathbf{S}\left(X_{1}, W\right)\right) \phi(g(e))=x\right\} .
$$

Further $g\left(W_{0}\right) \subseteq W_{1}$.

Proof. The equivalence of (i) and (ii) follows from Lemmas 3.10 and 3.11 and from Theorem 2.11 of Goldberg (1981). Condition (iii) is an alternative way of stating condition (ii). The final assertion follows from the fact that if $\phi \in \mathbf{S}(X, W)$ satisfies $\phi(e)=x$, then $\psi=\psi \uparrow_{X_{1}}$ is an S-morphism satisfying $\psi(g(e))=g(x)$. 
Before discussing cocatalytic objects in $\mathfrak{X}$ we give an example to illustrate Theorem 3.15. We take $(X ; g)$ as shown below.

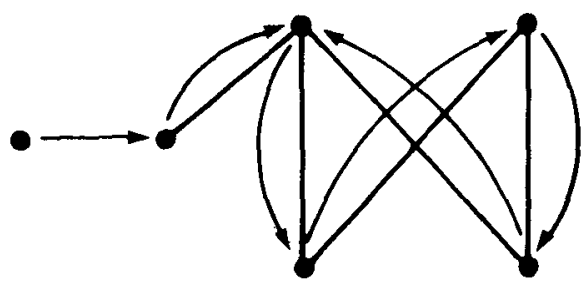

The set $\mathscr{P}$ consists of the following spaces. The accompanying inequalities are those satisfied by the distinguished end; these inequalities determine the associated $\sigma_{(Y, e)}$.

(1)

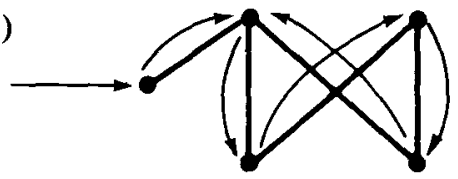

$g \leqslant g^{2}=g^{6} ; g^{2}, g^{4} \geqslant g^{3}, g^{5}$

(3)

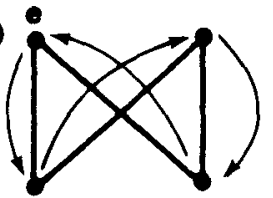

$1=g^{4} ; 1, g^{2} \geqslant g, g^{3}$

(7)

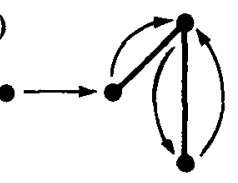

$g^{4}=g^{2} \geqslant g, g^{3}$
(4)

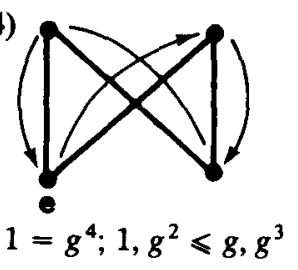

(8)

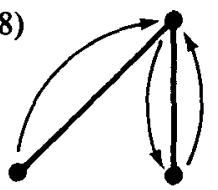

$1, g^{2} \leqslant g=g^{3}$
(2)

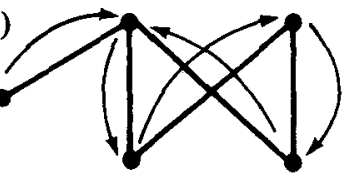

$1 \leqslant g=g^{5} ; g, g^{3} \geqslant g^{2}, g^{4}$

(5)

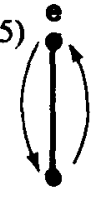

$1=g^{2} \geqslant g$

(6)

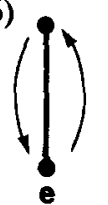

$1=g^{2} \leqslant g$

(11)
$\longrightarrow 0$
(12)

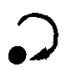
$g^{2}=g$
$g=1$ 
We therefore have $\mathscr{P}$ as shown

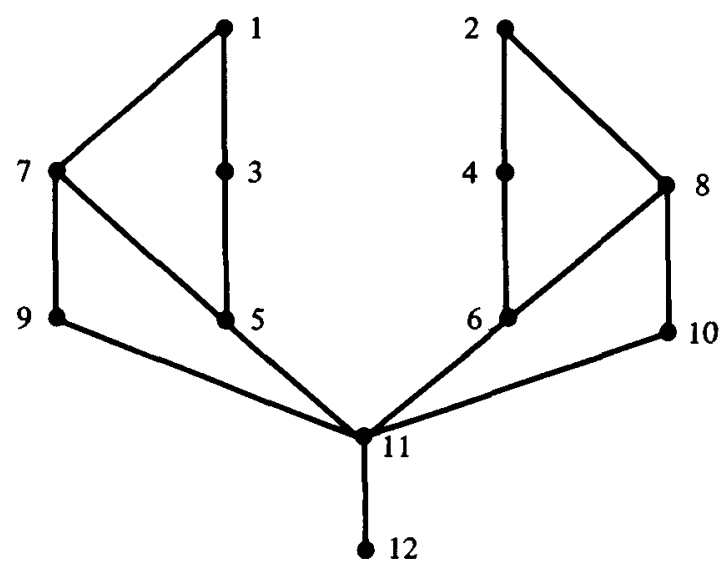

In this case any $E \in \mathfrak{X}$ is the union of

$$
W_{0}=\left\{x \in W \mid g^{6}(x)=g^{2}(x) \geqslant g(x) \text { and } g^{2}(x), g^{4}(x) \geqslant g^{3}(x), g^{5}(x)\right\}
$$
and

$$
W_{1}=\left\{x \in W \mid g^{5}(x)=g(x) \geqslant x \text { and } g(x), g^{3}(x) \geqslant g^{2}(x), g^{4}(x)\right\} \text {. }
$$

LeMmA 3.14. Let $\varnothing \neq W \in \operatorname{Cocat}(\mathfrak{X})$ and take $W_{0}$ and $W_{1}$ as in Theorem 3.13 . Then the g-map on $W$ is constant on each of $W_{0}$ and $W_{1}$ (and hence constant on $W$ whenever $W_{0}$ and $W_{1}$ have a non-empty intersection).

Proof. We have

$$
\mathbf{S}(X, W)=\left\{f_{x}: g^{k}(e) \mapsto g^{k}(x) \mid x \in W_{0}\right\}
$$

As in the proof of Theorem 3.9, $f_{x} \geqslant f_{y}$ if and only if $x \geqslant y$ and $g(x)=g(y)$. Since $\mathbf{S}(X, W)$ is a lattice, $g$ is constant on $W_{0}$. Similarly, consideration of

$$
\mathbf{S}\left(X_{1}, W\right)=\left\{f_{x}: g^{k}(g(e)) \mapsto g^{k}(x) \mid x \in W_{1}\right\}
$$

shows that $g$ is constant on $W_{1}$.

THEOREM 3.15. Suppose that $\underline{P}$ is a finite simple Ockham algebra, and let $\mathbf{K}=\mathbf{H S P}(\underline{P})$.

(i) If $\underline{P}$ is a Boolean lattice, then $\operatorname{Cat}(K)=\{\underline{1}, \underline{2}\}$;

(ii) If $\underline{P}$ is not a Boolean lattice, then $\operatorname{Cat}(K)=\{\underline{1}, \underline{2}, \underline{K}\}$, where $\underline{K}$ denotes the 3-element Kleene algebra.

Proof. As usual, let $X=D(\underline{P})$, and let $\mathfrak{X}=D(\mathbf{K})$. Whenever $W \in \mathfrak{X}$ and $|W| \leqslant 1$, then trivially $W \in \operatorname{Cocat}(\mathfrak{X})$, so that $\underline{1}$ and $\underline{2}$ are always catalytic in $K$. If $\underline{P}$ is not a Boolean lattice, then $X$ has height one, and $T$ belongs to $\operatorname{GM}(X)$. 
Consequently $T \in \mathfrak{X}$ in this case. Consider $\mathbf{S}(Y, T)$, where $Y \in \mathfrak{X}$. We fix an end $e$ of $X$ which we may assume to be a minimal point. We have that $Y$ is the union of $Y_{0}=Y_{(X, e)}$ and $Y_{1}=Y_{\left(X_{1}, g(e)\right)}$. Let $t$ be the minimal point in $T$. Let $\phi \in$ $\mathrm{S}(Y, T)$. For each $y \in Y, \phi(y)=t$ or $\phi(y)=g(t)$, and $\phi(y)$ satisfies all inequalities satisfied by $y$. It follows that $\phi$ maps $Y_{0}$ to $t$ and $Y_{1}$ to $g(t)$. Therefore $\mathbf{S}(Y, T)=\varnothing$ unless $Y_{0} \cap Y_{1}=\varnothing$, in which case $|\mathbf{S}(Y, T)|=1$. Hence $\mathrm{S}(Y, T)$ is a lattice. Thus $T \in \operatorname{Cocat}(\mathfrak{X})$. Equivalently $\underline{K} \in \operatorname{Cat}(\mathbf{K})$, as required.

The assumption that $\underline{P}$ is simple is equivalent to the assumption that $X$ is a loop. When $X$ is an antichain, it is clear that $W_{0}=W_{1}$. Hence Lemma 3.14 implies that $g$ is constant on $W$. Since, for some $k>0, g^{k}=1$ on $W$, we have $|W| \leqslant 1$. This completes the proof of (i).

Finally suppose that $X$ is not an antichain and that $W \in \operatorname{Cocat}(\mathfrak{X})$ is such that $|W|>1$. We must show that $W=T$. If $g$ were constant on $W$, the assumption that $X$ is a loop would force $|W| \leqslant 1$, contrary to hypothesis. Hence (by Lemma 3.14) $g$ takes two distinct values, one, $u$ say, on $W_{0}$, the other, $v=g(u)$ on $W_{1}$. Again because $g^{k}=1$ on $W$, each of $W_{0}$ and $W_{1}$ contains one point. The possibilities for $W$ are
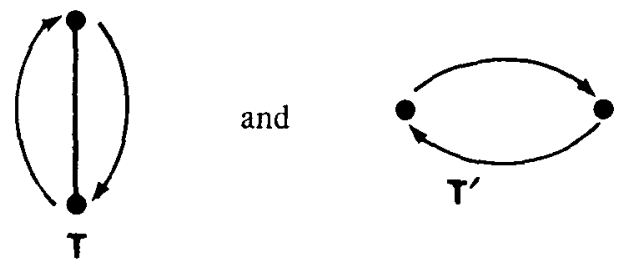

Clearly $T^{\prime}$ is not cocatalytic in any class containing it, since if $\phi \in \mathbf{S}\left(Y, T^{\prime}\right)$ then $\phi$ and $g \circ \phi$ have no supremum.

Note that we have proved that if $\mathbf{K}$ is the variety generated by a finite simple Ockham algebra which is not a Boolean lattice, then $K \in \mathbf{K}$, and for all $A \in \mathbf{K}$ we have $|\mathbf{K}(\underline{K}, A)| \leqslant 1$. Consequently each $A \in K$ has at most one -fixed point.

We finally consider what happens when $\underline{P}$ is not assumed to be simple. Suppose that $W$ is cocatalytic in the dual category $\mathfrak{X}$. We know that the $g$-map is constant on $W_{0}$ and $W_{1}$. As a result the defining inequalities for $W_{0}$ and $W_{1}$ given in Theorem 3.13 simplify. We may also have $W_{1} \subseteq W_{0}=W$; this happens in particular whenever $X$ satisfies one of (a)-(c) in Lemma 3.12.

Consider first the case when $g$ is constant. (This is forced to occur whenever $W_{1} \subseteq W_{0}=W$.) The possible forms for $W_{0}$ and $W_{1}$ are just

$$
W, \quad\{x \in W \mid x \leqslant u\}, \quad\{x \in W \mid x \geqslant u\} \text { and }\{u\}
$$

(where $g$ takes the constant value $u \in W_{1}$ ). 
Now suppose we have a 2-valued function $g$ taking values $u$ and $v$ on $W_{0}$ and $W_{1}$, respectively. Since $g\left(W_{0}\right) \subseteq W_{1}, g(u)=v$. Necessarily $g^{2}(u)=u$ or $v$. Suppose, by way of contradiction, that $g^{2}(u)=v$. Then $g(u)$ is fixed by $g$ and belongs both to $W_{0}$ and $W_{1}$, which would imply that $g$ is constant on $W$. Thus $g(v)=u$, whence $g^{2}(u)=u$ and $g^{2}(v)=v$. The defining inequalities for $W_{0}$ reduce to inequalities relating to a subset of $\left\{x, g(x)=u, g^{2}(x)=v\right\}$, where $x$ is a variable point of $W_{0}$, and similarly for $W_{1}$.

Let $W \in \operatorname{Cocat}(\mathfrak{X})$. It is easy to see in any specific case that Lemma 3.1 may be applied to show that $W_{0}$ and $W_{1}$ are topological lattices.

We thus have as candidate cocatalytic spaces in $\mathfrak{X}$ those $W$ for which the $g$-map is 1-valued or 2-valued, and $W_{0}$ and $W_{1}$ are topological lattices described by appropriate inequalities, as indicated above. Any such space $W$ is indeed cocatalytic. To see this, take $Y \in \mathfrak{X}$ and let $\phi, \psi \in \mathbf{S}(Y, W)$. Each of $\phi$ and $\psi$ maps $Y_{0}$ into $W_{0}$ and $Y_{1}$ into $W_{1}$. Since $W_{0}$ and $W_{1}$ are topological lattices, the pointwise supremum and infimum of $\phi$ and $\psi$ exist and are P-morphisms. They are S-morphisms because $g$ is constant on $W_{0}$ and $W_{1}$.

Rather than give a complicated general description of $\operatorname{Cocat}(\mathfrak{X})$, we conclude with some selected examples.

EXAMPLES. (1) Take $X$ to be

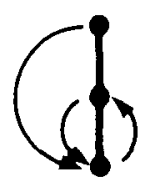

Then any $W \in \mathfrak{X}$ is the union of

$$
W_{0}=\left\{x \in W \mid x \geqslant g^{2}(x) \geqslant g(x)=g^{3}(x)\right\}
$$

and

$$
W_{1}=\left\{x \in W \mid x=g^{2}(x) \leqslant g(x)\right\} .
$$

Spaces $W$ with constant $g$-map taking value $u$ have $W_{0}=\{x \in W \mid x \geqslant u\} \supseteq$ $W_{1}=\{u\}$. If $g$ has values $u$ on $W_{0}$ and $v(\neq u)$ on $W_{1}$, then $W_{0}=\{x \in W \mid x \geqslant$ $v \geqslant u=g(x)\}$ and $W_{2}=\{v\}$. The non-empty cocatlytic spaces are of the form shown below; $u$ and $v$ may, but need not, coincide, and the whole space is a topological lattice.

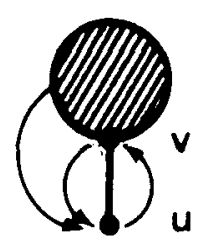


(2) Take $X$ to be

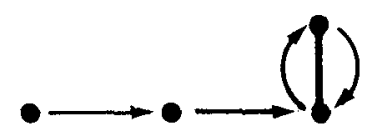

Here $W \in \mathfrak{X}$ is the union of

$$
W_{0}=\left\{x \in W \mid g^{2}(x)=g^{4}(x) \leqslant g^{3}(x)\right\}
$$

and

$$
W_{1}=\left\{x \in W \mid g(x)=g^{3}(x) \leqslant g^{2}(x)\right\} .
$$

A constant $g$-map with any image is admissible, while the only restriction on a $g$-map taking distinct values $u$ on $W_{0}$ and $v$ on $W_{1}$ is that $v<u$. Lemma 3.1 can be used to show that $W$ and $W_{1}$ must be topological lattices if $W$ is to be cocatalytic.

Let $W$ be a member of $\mathbf{P}$ which is a disjoint union of clopen topological lattices $W_{0}$ and $W_{1}$ such that no element of $W_{0}$ dominates an element of $W_{1}$, and at least one element of $W_{0}$, say $u$, is dominated by an element, say $v$, of $W_{1}$. Let $g$ map $W_{0}$ to $u$ and $W_{1}$ to $v$. Then $W \in \mathfrak{X}$, and the remarks preceding these examples imply that it is cocatalytic. The only cocatalytic spaces not of this type are topological lattices with constant $g$-map. The simplest example of a space which is cocatalytic in $\mathfrak{X}$ but is not a topological lattice is given below:

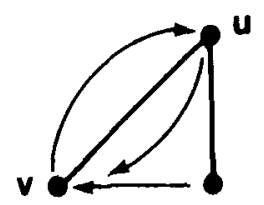

We can now substantiate the claim made at the beginning of this section. The example above shows that there exist varieties of distributive-lattice-ordered algebras containing catalytic objects which are not catalytic in D. On the other hand, there exist lattices $A \in \mathrm{Cat}(\mathrm{D})$ which have an operation of negation which makes them into Kleene algebras but which are not catelytic as Kleene algebras. To see this, take $A$ to be the linear sum of $B \in \operatorname{Cat}(D)$ and its order-theoretic dual $B^{d}$ and let $\sim$ be the obvious dual lattice-isomorphism of $A$. Then $A$ is a Kleene algebra and $A \in \operatorname{Cat}(\mathbf{D})$, but for $|B| \geqslant 2, A$ is not a catalytic Kleene algebra, by Theorem 3.15(ii). Further light is shed on the behaviour of Kleene algebras by Theorems 5.2-5.4 and the discussion of colattices in Section 6 .

The table below summarises the process of characterising catalytic objects in the most familiar varieties. The notation is that of Lemmas 3.1 and 3.2. We write $\underline{k}$ for the $k$-element chain with the discrete topology. 


\begin{tabular}{|c|c|c|c|c|c|c|c|c|c|}
\hline 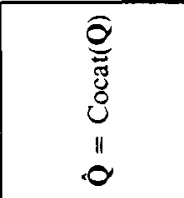 & 3 & & & $\frac{\mathscr{J}}{\nu}$ & $\ddot{\nu}$ & & & $\stackrel{\ddot{x}}{\grave{\nu}}$ & $\frac{\tilde{3}}{2}$ \\
\hline 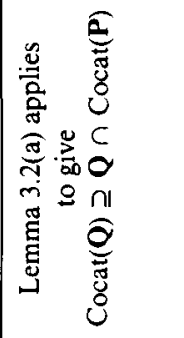 & & $\mathscr{E}$ & $\nu_{\nu}^{2}$ & & & $\mid \begin{array}{c}8 \\
2 \\
21\end{array}$ & $\stackrel{\ddot{\nu}}{\nu}$ & & \\
\hline 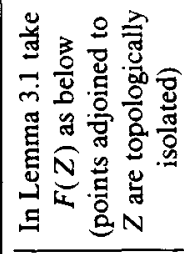 & & $\begin{array}{l}\vec{\oplus} \\
\stackrel{N}{N}\end{array}$ & $\begin{array}{l}\vec{\oplus} \\
N \\
\oplus \\
-\end{array}$ & & & 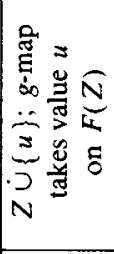 & 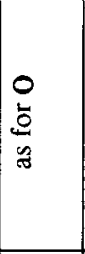 & & \\
\hline 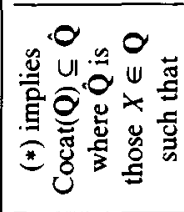 & $\frac{\vec{v}}{\underline{x}}$ & $\begin{array}{l}\vec{v} \\
\frac{v}{x} \\
x \\
\underline{x} \\
\underline{E}\end{array}$ & 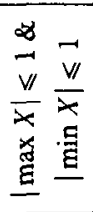 & 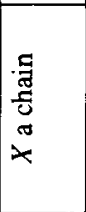 & 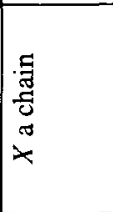 & 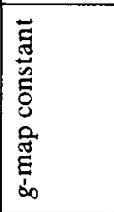 & 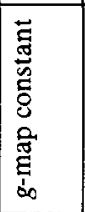 & $\begin{array}{l}\vec{v} \\
\underline{\underline{x}} \\
\underline{x}\end{array}$ & $\frac{v}{v}$ \\
\hline 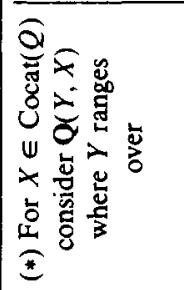 & $\rightarrow 1$ & $\rightarrow$ & $N$ & \begin{tabular}{c}
7 \\
1 \\
$\vdots$ \\
$\vdots$ \\
$\vdots$ \\
$\vdots$ \\
\hdashline$i$
\end{tabular} & 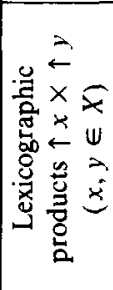 & zo & $E$ & $\Xi$ & h \\
\hline 10 & 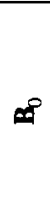 & $\begin{array}{r}3 \\
3 \\
v= \\
= \\
V \\
=\end{array}$ & 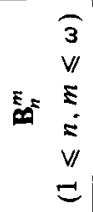 & $\begin{array}{r}\hat{3} \\
=v \\
= \\
v \\
c \\
c\end{array}$ & $3^{3}$ & 0 & 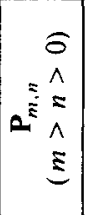 & 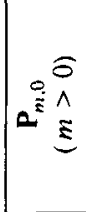 & 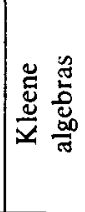 \\
\hline
\end{tabular}




\section{Ordered hom-sets and duality}

The method employed in Section 3 for locating catalytic objects relied on the order-isomorphism between ordered hom-sets which enables us to transform our original problem to a dual one in a dual category. The duality we used was the restriction to a given subcategory $\mathbf{C}$ of $\mathbf{D}$ of the $\mathbf{D}-\mathbf{P}$ duality (for which Lemma 2.2 provided the necessary order-isomorphism). For some varieties of distributive-lattice-ordered algebras more intrinsic dualities are available. In their paper Davey and Werner (1983a) gave a general scheme for creating dualities for certain classes of algebras. Their approach yields, among others, dualities for Stone and double Stone algebras, de Morgan, Kleene and Ockham algebras. In each of these cases, the duality is based on a schizophrenic object $P$ which carries an order relation as an object of the algebraic category and a different order relation as a member of the dual category, and the objects in both categories are ordered. (For example, for Stone algebras, $P$ is the 3-element chain with orders:

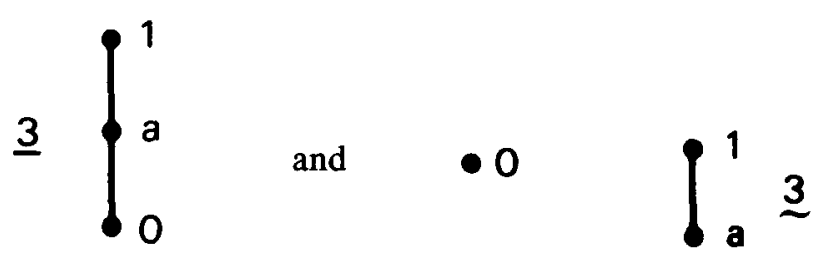

(see Davey (1978)(1982), Davey and Werner (1983a)).) In such a situation, it is natural to seek conditions under which the bijection between hom-sets provided by the adjunction establishing the duality is an order-isomorphism, and to investigate the order structure of the hom-sets more generally.

This section is devoid of examples, but it can be read in parallel with Section 5 which illustrates the theory developed here.

Following Davey and Werner (1983a), we shall assume that $\mathbf{K}=\mathbb{I S P}(\underline{P})$ is the prevariety generated by some algebra $\underline{P}=(P ; F)$, where $F$ is a set of finitary operations on $P$, and $P$ has a compact Hausdorff topology $\tau$ with respect to which all the operations in $F$ are continuous. We also assume that $\underset{\sim}{P}=(P$; $\tau, G, R$ ) is a topological structure (on the same underlying set $P$ ), where

(a) each $g \in G$ is a K-homomorphism $g: \underline{P}^{n} \rightarrow \underline{P}$ for some $n \geqslant 0$

(b) each $r \in R$ is a subalgebra of $\underline{P}^{n}$ for some $n \geqslant 1$. 
When $P$ is finite we suppress $\tau$, which is necessarily discrete. We define $\mathfrak{X}=\operatorname{EP}(\underset{\sim}{P})$ to be the class of all structures which embed as closed topological substructures of a power of $P$. The morphisms of the category $\mathfrak{X}$ are, of course, all continuous structure-preserving maps.

The set $P$ sits in $\mathbf{K}$ as $\underline{P}$ and in $\mathfrak{X}$ as $\underset{\sim}{P}$. In most cases of interest to us, $P$ is finite. However, $P$ may be infinite: for example, when $\mathbf{K}$ is the variety of Ockham algebras, $P=2^{N}$. We could also have allowed partial operations on $P$; we have not done so because these are not needed in any of our examples.

The compatibility relations (a) and (b) guarantee that the natural contravariant hom-functors

$$
D: \mathbf{K} \rightarrow \mathfrak{X}, \quad \text { where } D(A):=\mathbf{K}(A, \underline{P}) \leqslant \underline{P}^{A}
$$

and

$$
E: \mathfrak{X} \rightarrow \mathbf{K} \text {, where } E(X):=\mathfrak{X}(X, \underset{\sim}{P}) \leqslant \underline{P}^{X}
$$

are well-defined (and adjoint on the right); see Davey and Werner (1983a). We shall assume that, in the terminology of Davey and Werner (1983a), $D$ and $E$ give a full duality between $K$ and $\mathfrak{X}$; more specifically, we assume that the natural evaluation maps $e_{A}$ and $\varepsilon_{X}$ are isomorphisms for each $A \in \mathbf{K}$ and each $X \in \mathfrak{X}$. Here $e_{A}: A \rightarrow E D(A)=\mathfrak{X}(\mathbf{K}(A, \underline{P}), \underline{P})$ is given by $e_{A}(a)(g):=g(a)$ for all $g \in \mathbf{K}(A, \underline{P})$ and all $a \in A$, and $\varepsilon_{X}: X \rightarrow D E(X)=\mathbf{K}(\mathfrak{X}(X, \underline{P}), \underline{P})$ is given by $\varepsilon_{X}(x)(\phi):=\phi(x)$ for all $\phi \in \mathfrak{X}(X, P)$ and all $x \in X$. This is exactly the situation which prevails for the D-P duality.

We now suppose that the algebras in $\mathbf{K}$ carry a natural order (as happens, for example, when they are lattices with some extra operations). We denote the order relation in each $A \in \mathbf{K}$ by $\leqslant$. Order relations, which we again denote by $\leqslant$, are induced on hom-sets as follows:

(O1) for any $A, B \in \mathbf{K}, \mathbf{K}(A, B)$ is ordered pointwise from $B$;

(O2) for every $X \in \mathfrak{X}, \mathfrak{X}(X, \underset{\sim}{P})$, as a subset of $P^{X}$, inherits the pointwise extension of the order $\leqslant$ on $\underline{P}$.

At this stage we are not assuming any intrinsic order on the objects of $\mathfrak{X}$; all the order structure comes from $\mathbf{K}$. Obviously we should like all $\mathbf{K}$-homomorphisms to be $\leqslant$-preserving. The following proposition gives criteria for this; $(\mathrm{O} 2)$ is involved but not $(\mathrm{O} 1)$.

Proposition 4.1. The following are equivalent:

(i) every $\mathbf{K}$-homomorphism is $\leqslant$-preserving;

(ii) for each $A \in \mathbf{K}$, the evaluation map $e_{A}: A \rightarrow E D(A)$ is a $\leqslant$-isomorphism;

(iii) for each $A \in \mathbf{K}$, every homomorphism $\mathrm{g} \in \mathbf{K}(A, \underline{P})$

is $\leqslant$-preserving and, whenever $a, b \in A$ with $a \nless b$, there exists $g \in \mathbf{K}(A, \underline{P})$ with $g(a) \nless g(b)$. 
Proof. (i) $\Rightarrow$ (ii) is trivial.

(ii) $\Rightarrow$ (iii). Let $a, b \in A$ satisfy $a \leqslant b$. By (ii), $e_{A}(a) \leqslant e_{A}(b)$ in $\mathfrak{X}(\mathbf{K}(A, \underline{P}), \underline{P})$. This means that, for $g \in \mathbf{K}(A, \underline{P}), e_{A}(a)(g) \leqslant e_{A}(b)(g)$ (from (O2)); that is, $g(a) \leqslant g(b)$. Hence every $g \in \mathbf{K}(A, \underline{P})$ is order-preserving.

Now suppose $a, b \in A$ are such that $a \nless b$. Then $e_{A}(a) \nless e_{A}(b)$ by (ii). Hence there exists $g \in \mathbf{K}(A, \underline{P})$ with $g(a)=e_{A}(a)(g) \nless e_{A}(b)(g)=g(b)$.

(iii) $\Rightarrow$ (ii) is proved by reversing the argument above.

(ii) $\Rightarrow$ (i). Let $h: A \rightarrow B$ be a $\mathbf{K}$-homomorphism and consider the diagram below

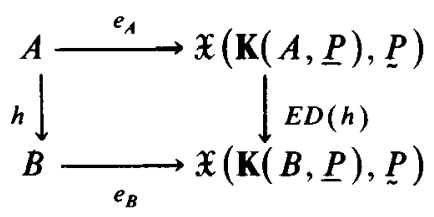

By (ii), to show that $h$ preserves $\leqslant$, it is enough to show that $E D(h)$ does, and this is routine to check.

Dually, we may suppose that the objects in $\mathfrak{X}$ carry an order relation $\preccurlyeq$ and induce order relations, again denoted by $\preccurlyeq$, on hom-sets as follows:

$(\mathrm{O} 1)^{*}$ for every $X, Y \in \mathbf{K}, \mathfrak{X}(X, Y)$ is ordered pointwise from $Y$;

$(\mathrm{O} 2)^{*}$ for every $A \in \mathbf{K}, \mathbf{K}(A, \underline{P})$, as a subset of $P^{A}$, inherits the pointwise extension of the order $\preccurlyeq$ on $\underset{\sim}{P}$.

A dual version of Proposition 4.1 gives criteria for $\mathfrak{X}$-morphisms to be $\leqslant$ preserving. Obviously every $\mathfrak{X}$-morphism preserves $\leqslant$ if $\leqslant$ on $\underset{\sim}{P}=(P ; \tau, G, R)$ belongs to $R$. We henceforth assume that the objects of both $\mathbf{K}$ and $\mathfrak{X}$ are ordered. The hom-sets $\mathbf{K}(A, \underline{P})(A \in \mathbf{K})$ now carry two order relations: $\leqslant$ (given by (O1) and independent of the duality) and $\leqslant$ (given by $(\mathrm{O} 2)^{*}$, through the duality). In general these orders do not coincide. (See Proposition 4.14 for the condition under which they do coincide.) Similarly, each $\mathfrak{X}(X, \underset{\sim}{P})(X \in \mathfrak{X})$ carries order relations $\leqslant$ (given by $\left.(\mathrm{O} 1)^{*}\right)$ and $\leqslant$ (given by $(\mathrm{O} 2)$ ). For general hom-sets $\mathbf{K}(A, B)$ and $\mathfrak{X}(X, Y)$, only a single order relation (respectively, $\leqslant$ from $(\mathrm{O} 1)$ and $\preccurlyeq$ from $\left.(\mathrm{O} 1)^{*}\right)$ is available.

Theorem 4.2. Let $A, B \in \mathbf{K}$. Then $(\mathbf{K}(A, B) ; \leqslant)$ and $(\mathfrak{X}(D(B), D(A)) ; \preccurlyeq)$ are order-isomorphic via the natural bijection, provided that

(i) K-homomorphisms into $\underline{P}$ are $\leqslant-$ preserving;

(ii) $A$ is such that (H1) for $\mathrm{g}, h \in \mathbf{K}(A, \underline{P}), \mathrm{g} \leqslant h$ implies that $\mathrm{g} \preccurlyeq h$;

(iii) $B$ is such that

(H2) for $a, b \in B, a \nless b$ implies that $g(a) \star g(b)$

(in $\underset{\sim}{P}$ ) for some $g \in \mathbf{K}(B, \underline{P})$. 
Proof. The natural bijection between $\mathbf{K}(A, B)$ and $\mathfrak{X}(D(B), D(A))$ is just the functor $D$ restricted to the set $\mathbf{K}(A, B)$. Assume (i), (ii) and (iii) hold. By definition, if $h \leqslant k$ in $\mathbf{K}(A, B)$, then, for all $a \in A$, we have $h(a) \leqslant k(a)$ in $B$. Since K-homomorphisms into $\underline{P}$ preserve $\leqslant$, it follows that, for all $g \in \mathbf{K}(B, \underline{P})$, we have $(g \circ h)(a) \leqslant(g \circ k)(a)$. Hence $g \circ h \leqslant g \circ k$ in $\mathbf{K}(A, P)$. By (ii), $g \circ h \leqslant$ $g \circ k$ for all $g \in \mathbf{K}(B, \underline{P})$; that is, $D(h) \leqslant D(k)$. We have thus shown that $D$ : $(\mathbf{K}(A, B) ; \leqslant) \rightarrow(\mathfrak{X}(D(B), D(Z)) ; \preccurlyeq)$ is order-preserving.

Assume now that $D(h) \leqslant D(k)$ in $\mathfrak{X}(D(B), D(A))$, so that, for all $g \in D(B)$ $=\mathbf{K}(B, \underline{P}), g \circ h \preccurlyeq g \circ k$ in $D(A)=\mathbf{K}(A, \underline{P})$. Then $g(h(a)) \preccurlyeq g(k(a))$ for all $a \in A$ and for all $g \in \mathbf{K}(B, \underline{P})$. It follows from (iii) that $h(a) \leqslant k(a)$ for all $a \in A$; that is, $h \leqslant k$ in $\mathbf{K}(A, B)$, as required.

We have given sufficient conditions for the isomorphism of the hom-sets $\mathbf{K}(A, B)$ and $\mathfrak{X}(D(B), D(A))$. We should like to know the extent to which these conditions are necessary. To discuss this fully we need to consider the effect of imposing further compatibility conditions.

The compatibility conditions (a) and (b) on the algebra $\underline{P}$ and the structure $\underline{P}$ already guarantee that the natural map

$$
\rho: D(F \mathbf{K}(1))=\mathbf{K}(F \mathbf{K}(1), \underline{P}) \rightarrow \underset{\sim}{P}
$$

given by $\rho(g)=g\left(x_{1}\right)$, where $x_{1}$ is the free generator of $F \mathbf{K}(1)$, is an isomorphism in $\mathfrak{X}$. If $\leqslant$ belongs to $R$, then $\rho$ is trivially a $\preccurlyeq$-isomorphism. More generally we have

Proposition 4.3. The map $\rho: D(F \mathbf{K}(1)) \rightarrow \underset{\sim}{P}$ is $a \leqslant$-isomorphism if and only if (F) $a \leqslant b$ in $P$ implies that $p(a) \preccurlyeq p(b)$ for all unary term functions $p$ on the algebra $\underline{P}$. In particular, $\rho$ is $a \leqslant-i$ isomorphism if $\leqslant$ is algebraic over $\underline{P}$ (that is, if $\preccurlyeq$ is a subalgebra of $\underline{P}^{2}$ ).

Proof. Assume $\rho$ is a $\preccurlyeq$-isomorphism and suppose that $a \leqslant b$ in $P$. Let $k_{a}$ and $k_{b}$ be the homomorphisms in $\mathbf{K}(F \mathbf{K}(1), \underline{P})$ determined by $k_{a}\left(x_{1}\right)=a$ and $k_{b}\left(x_{1}\right)=b$. Then

$$
\rho\left(k_{a}\right)=k_{a}\left(x_{1}\right)=a \preccurlyeq b=k_{b}\left(x_{1}\right)=\rho\left(k_{b}\right),
$$

and hence $k_{a} \leqslant k_{b}$. For any unary term $p\left(x_{1}\right)$, we have $k_{a}\left(p\left(x_{1}\right)\right) \leqslant k_{b}\left(p\left(x_{1}\right)\right)$, and so $p(a) \leqslant p(b)$, as required.

Conversely assume that (F) holds. If $g \leqslant h$ in $\mathbf{K}(F \mathbf{K}(1), \underline{P})$, then by definition we have $\rho(g)=g\left(x_{1}\right) \leqslant h\left(x_{1}\right)=\rho(h)$. Assume $\rho(g) \leqslant \rho(h)$; that is, $g\left(x_{1}\right) \leqslant$ $h\left(x_{1}\right)$. Let $p\left(x_{1}\right)$ be a unary term. By (F), $p\left(g\left(x_{1}\right)\right) \preccurlyeq p\left(h\left(x_{1}\right)\right)$, and so $g\left(p\left(x_{1}\right)\right)$ $\leqslant h\left(p\left(x_{1}\right)\right)$. Thus $g \leqslant h$ in $\mathbf{K}(F \mathbf{K}(1), \underline{P})$.

It is trivial that $(\mathrm{F})$ holds if $\leqslant$ is a subalgebra of $\underline{P}^{2}$. 
We can now give sufficient conditions for $(\mathrm{H} 1)$ and $(\mathrm{H} 2)$ to hold for a specific algebra $A$.

Proposition 4.4. (i) If the natural bijection from $(\mathbf{K}(A, P) ; \leqslant)$ to $(\mathfrak{X}(D(\underline{P}), D(A)) ; \preccurlyeq)$ is an order-isomorphism, then $(\mathrm{H} 1)$ holds for $A$.

(ii) Assume that $(F)$ holds. If the natural bijection from $(\mathbf{K}(F \mathbf{K}(1), B) ; \leqslant)$ to $(\mathfrak{X}(D(B), D(F \mathbf{K}(1))) ; \preccurlyeq)$ is an order isomorphism, then $(\mathrm{H} 2)$ holds for $B$.

Proof. (i) Let $g, h \in \mathbf{K}(A, \underline{P})$ satisfy $f \leqslant h$. By hypothesis, $D(g) \leqslant D(h)$ in $\mathfrak{X}(D(\underline{P}), D(A))$. Hence, for every endomorphism $e$ of $\underline{P}$, we have $e \circ g \preccurlyeq e \circ h$ in $D(A)=\mathbf{K}(A, \underline{P})$; in particular, $g=\mathrm{id} \circ g \preccurlyeq \mathrm{id} \circ h=h$, where id denotes the identity map on $\underline{P}$.

(ii) Suppose $a, b \in B$ are such that $g(a) \preccurlyeq g(b)$ for all $g \in \mathbf{K}(B, \underline{P})$. Let $k_{a}$, $k_{b} \in \mathbf{K}(F \mathbf{K}(1), B)$ be determined by $k_{a}\left(x_{1}\right)=a$ and $k_{a}\left(x_{1}\right)=b$. Then $\left(g \circ k_{a}\right)\left(x_{1}\right) \preccurlyeq\left(g \circ k_{b}\right)\left(x_{1}\right)$ in $\underset{P}{P}$ for all $g \in \mathbf{K}(B, \underline{P})$. Hence $g \circ k_{a} \leqslant g \circ k_{b}$ in $\mathbf{K}(F \mathbf{K}(1), \underline{P})$ for all $g \in \mathbf{K}(B, \underline{P})$, by Proposition 4.3. This implies that $D\left(k_{a}\right) \leqslant$ $D\left(k_{b}\right)$ and hence, by hypotheses, that $k_{a} \leqslant k_{b}$. Thus $a=k_{a}\left(x_{1}\right) \leqslant k_{b}\left(x_{1}\right)=b$.

Combining Theorem 4.2 and Proposition 4.4 we have

THEOREM 4.5. Suppose that $\mathbf{K}$-homomorphisms into $\underline{P}$ are $\leqslant-$ preserving and that (F) holds. Let $A, B \in \mathbf{K}$. Then the following are equivalent.

(I) The natural bijection between $(\mathrm{K}(A, B)$; $\leqslant)$ and $(\mathfrak{X}(D(B), D(A)) ; \leqslant)$ is an order isomorphism.

(II) The natural bijections between $(\mathrm{K}(A, \underline{P}) ; \leqslant)$ and $(\mathfrak{X}(D(\underline{P}), D(A)) ; \preccurlyeq)$, and between $(\mathbf{K}(F \mathbf{K}(1), B) ; \leqslant)$ and $(\mathfrak{X}(D(B), D(F \mathbf{K}(1))) ; \preccurlyeq)$, are order-isomorphisms.

(III) (H1) holds for $A$ and (H2) holds for $B$.

Almost all the preceding results have, mutatis mutandis, dual versions. The only difficulty arises in connection with Proposition 4.3, for which we need to consider $F \mathfrak{X}(1)$ in place of $F \mathbf{K}(1)$. It is the case that $\underline{P}$ is isomorphic to $E(F \mathfrak{X}(1))$. However, to dualise the sufficiency part of Proposition 4.3, we require moreover that the elements of $F \mathfrak{X}(1)$ should be given by terms. We can prove the following result covering the finite case.

LemMa 4.6. Let $P=(P ; G, R)$, where $P$ is finite. Then the free 1-generated object in $\mathfrak{X}$ is the free 1-generated object $F_{1}$ in $\mathfrak{X}_{0}=\mathbb{1 S P}((P ; G))$; the topology on $F_{1}$ is discrete, and an $n$-ary relation $r$ in $R$ is given on $F_{1}$ by

$$
\left(\forall t_{1}, \ldots, t_{n} \in F_{1}\right)\left[\left(t_{1}, \ldots, t_{n}\right) \in r_{F_{1}} \Leftrightarrow(\forall a \in P)\left(t_{1}(a), \ldots, t_{n}(a)\right) \in r_{P}\right] .
$$


Proof. We first show that $F_{1}$, with the relations defined above, is an object in $\mathfrak{X}$. Let $\mu: F_{1} \rightarrow Z:=P^{P}$ be the natural $\mathfrak{X}_{0}$-embedding, mapping $t \in F_{1}$ to the corresponding term function on $P$. Now

$$
\begin{aligned}
\left(\mu\left(t_{1}\right), \ldots, \mu\left(t_{n}\right)\right) \in r_{Z} & \Leftrightarrow(\forall a \in P)\left(\mu\left(t_{1}\right)(a), \ldots, \mu\left(t_{n}\right)(a)\right) \in r_{P} \\
& \Leftrightarrow(\forall a \in P)\left(t_{1}(a), \ldots, t_{n}(a)\right) \in r_{P} \\
& \Leftrightarrow\left(t_{1}, \ldots, t_{n}\right) \in r_{F_{1}} .
\end{aligned}
$$

Hence $F_{1} \in \mathbb{E P}(\underline{P})=\mathfrak{X}$.

The proof is completed by showing that if $(\forall a \in P)\left(t_{1}(a), \ldots, t_{n}(a)\right) \in r_{\underline{P}}$, then

$$
(\forall X \in \mathfrak{X})(\forall x \in X)\left(t_{1}(x), \ldots, t_{n}(x)\right) \in r_{X},
$$

where $t_{1}, \ldots, t_{n}$ are unary term functions on $\underset{P}{ }$.

Let $X \leqslant \underline{P}^{S}$ and let $x \in X$. Then

$$
\begin{aligned}
\left(t_{1}(x), \ldots, t_{n}(x)\right) \in r_{X} & \Leftrightarrow(\forall s \in S)\left(t_{1}(x)(s), \ldots, t_{n}(x)(s)\right) \in r_{P} \\
& \Leftrightarrow(\forall s \in S)\left(t_{1}(x(s)), \ldots, t_{n}(x(s))\right) \in r_{\underline{P}} .
\end{aligned}
$$

The dual of Theorem 4.2 is of particular interest and is stated explicitly for ease of reference. It is proved by dualizing the proof of Theorem 4.2 and making use of the dual of Proposition 4.1.

Theorem 4.7. Let $A, B \in \mathbf{K}$. Then $(K(A, B) ; \leqslant)$ and $(\mathfrak{X}(D(B), D(A)) ; \preccurlyeq)$ are order-isomorphic via the natural bijection, provided that

(i) $\mathfrak{X}$-morphisms into $\underset{\sim}{P}$ are $\leqslant$-preserving;

(ii) $Y=D(B)$ is such that

$(\mathrm{H} 1)^{*}$ for $\phi, \psi \in \mathfrak{X}(Y, P), \phi \leqslant \psi$ implies $\phi \leqslant \psi$,

(iii) $X=D(A)$ is such that

$(\mathrm{H} 2)^{*}$ for $x, y \in X, x \leqslant y$ implies $\phi(x) \nless \phi(y)$

(in $\underline{P}$ ) for some $\phi \in \mathfrak{X}(X, \underline{P})$.

We now have two pairs of conditions: (H1) and (H2) (stated in Theorem 4.2) and $(\mathrm{H} 1)^{*}$ and $(\mathrm{H} 2)^{*}$ (stated in Theorem 4.7). These conditions are related as the following proposition shows. The proof is straightforward and is omitted.

Proposition 4.8. Suppose that $\mathbf{K}$-homomorphisms preserve $\leqslant$ and that $\mathfrak{X}$-morphisms preserve $\leqslant$. Then

(i) (H1) holds for $A \in \mathbf{K}$ if and only if (H2)* holds for $D(A)$; 
(ii) (H2) holds for $\boldsymbol{B} \in \mathbf{K}$ if and only if

$(\mathrm{H} 1)^{*}$ holds for $D(B)$.

So long as all morphisms are order-preserving, to show that $\mathbf{K}(A, B)$ is order-isomorphic to $\mathfrak{X}(D(B), D(A))$, it is thus sufficient to show that (H1) holds for $A$ and that $(\mathrm{H} 1)^{*}$ holds for $D(B)$. Generally we shall be interested in whether these conditions hold for all $A, B \in \mathbf{K}$. The following lemmas make checking extremely easy in practive. We prove only Lemma 4.9 . Lemma 4.10 is proved in much the same way (with the aid of Lemma 4.6).

LEMMa 4.9. The following conditions are equivalent:

(i) (H1) holds for all $A \in \mathbf{K}$;

(ii) (H2) holds for $A=F \mathbf{K}(1)$;

(iii) given $a, b \in P, a \neq b$ in $\underset{\sim}{P}$ implies that $p(a) \nless p(b)$ for some unary term function $p$ on $\underline{P}$.

Proof. It is trivial that (i) implies (ii). Now suppose that (ii) holds and assume that $a * b$ in $\underset{P}{\text {. Let }} k_{a}, k_{b} \in \mathbf{K}(F \mathbf{K}(1), \underline{P})$ be determined by $k_{a}\left(x_{1}\right)=a$, $k_{b}\left(x_{1}\right)=b$, where $x_{1}$ is the free generator of $F \mathbf{K}(1)$. Then $k_{a} * k_{b}$, so that by (ii), $k_{a} \nless k_{b}$. Hence there exists $c \in F \mathbf{K}(1)$ such that $k_{a}(c) \nless k_{b}(c)$. For some unary term $p$ we have $c=p\left(x_{1}\right)$, and so $p(a)=k_{a}\left(p\left(x_{1}\right)\right) \nless k_{b}\left(p\left(x_{1}\right)\right)=p(b)$. Thus (iii) holds.

Finally, we show that (iii) implies (i). Let $A \in \mathbf{K}$ and suppose that $g \nless h$ in $\mathbf{K}(A, \underline{P})$. For some $c \in A, g(c) \notin h(c)$. Using (iii) we can find a unary term $p$ such that $p(g(c)) \nless p(h(c))$. But then $g(p(c)) \nless h(p(c))$, and so $g \nless h$.

LEMMA 4.10. If $P$ is finite, the following conditions are equivalent. In general, (i) and (ii) are equivalent and implied by (iii):

(i) (H1)* holds for all $X \in \mathfrak{X}$;

(ii) (H1)* holds for $X=F \mathfrak{X}(1)$;

(iii) given $x, y \in P, x \nless y$ implies that $t(x) \nless t(y)$ for some unary term function ton $\underset{P}{ }$.

In summary, we have

THEOREM 4.11. Suppose that

(0) K-homomorphisms are $\leqslant$-preserving and $\mathfrak{X}$-morphisms are $\preccurlyeq$-preserving. 
If $P$ is finite, the following conditions are equivalent. In general, (I), (II), and (II)* are equivalent and implied by (III).

(I) For all $A, B \in \mathbf{K},(\mathbf{K}(A, B)$; $\leqslant)$ is order-isomorphic to $(\mathfrak{X}(D(B), D(A)) ; \preccurlyeq)$ via the natural bijection.

(II) (H1) For each $A \in \mathbf{K}, \leqslant \subseteq \leqslant$ on $\mathbf{K}(A, \underline{P})$. (H1)* For each $X \in \mathfrak{X}, \leqslant \subseteq \leqslant$ on $\mathfrak{X}(X, \underset{\sim}{P})$.

(II)* (H2) For each $A \in \mathbf{K}, a \notin b$ in $A$ implies that there exists $g \in \mathbf{K}(A, \underline{P})$ with $g(a) \vDash g(b)$ in $P$.

(H2)* For each $X \in \mathfrak{X}, x \neq y$ in $X$ implies that there exists $\phi \in \mathfrak{X}(X, \underline{P})$ with $\phi(x) \notin \phi(y)$ in $P$.

(II)(a) Given $a, b \in \underline{P}, a * b$ in $\underset{\sim}{P}$ implies that there exists $a$ unary term function $p$ on $\underline{P}$ such that $p(a) \notin p(b)$.

(b) Given $x, y \in \underline{P}, x \notin y$ in $\underline{P}$ implies that there exists a unary term function $t$ on $P$ such that $t(x) \star t(y)$.

RemarK 4.12. As noted earlier, $(0)$ holds whenever $\mathbf{K}$ is a variety of latticeordered algebras, and $\preccurlyeq$ on $\underset{P}{P}=(P ; \tau, G, R)$ belongs to $R$. Given that ( 0$)$ does hold, (II)(a) is automatically satisfied whenever $\leqslant \subseteq \preccurlyeq$ on $P$, while (II)(b) holds whenever $\leqslant \subseteq \leqslant$ on $P$.

We give one further general criterion for the satisfaction of $(\mathrm{H} 1),(\mathrm{H} 2),(\mathrm{H} 1)^{*}$, and $(\mathrm{H} 2)^{*}$. The motivation for this approach is the theory of piggyback dualities developed in Davey (1982) and generalized in Davey and Werner (1983b), (1985).

If the algebra $\underline{P}$ has a reduct in $\mathbf{D}$, then $\mathbf{K}=\mathbf{I S P}(\underline{P})$ becomes a class of bounded-distributive-lattice-ordered algebras. Similarly, if $(P ; \tau, \preccurlyeq)$ is an object of $\mathbf{P}$ (which holds automatically if $P$ is finite), and if $\mathfrak{X}$-morphisms preserve $\preccurlyeq$, then an easy application of the dual of Proposition 4.1 shows that $(X ; \tau, \preccurlyeq)$ is an object of $\mathbf{P}$ for all $(X ; \tau, G, R)$ in $\mathfrak{X}$. This shows that the maps $\Phi$ and $\Phi^{*}$ in the following proposition are well defined.

Proposition 4.13. (i) Assume that $\underline{P}$ has a reduct in $\mathbf{D}$ and that the order $\leqslant$ on each $A$ in $\mathbf{K}$ is the induced lattice order. If there is a map $\alpha: P \rightarrow 2$ which is a D-homomorphism and preserves $\leqslant$, and if the induced map

$$
\Phi=\alpha \circ-: \mathbf{K}(B, \underline{P}) \rightarrow \mathbf{D}(B, \underline{2})
$$

is onto, then (H2) holds for $B$.

(ii) Assume that $(P ; \tau, \preccurlyeq)$ is in $\mathbf{P}$ and that $\mathfrak{X}$-morphisms preserve $\preccurlyeq$. If there is a map $\alpha: P \rightarrow 2$ which is a $\mathbf{P}$-morphism and preserves $\leqslant$, and if the induced map

$$
\Phi^{*}=\alpha \circ-: \mathfrak{X}(X, \underline{P}) \rightarrow \mathbf{P}(X, \underset{\sim}{2})
$$

is onto, then $(\mathrm{H} 2)^{*}$ holds for $X$. 
Proof. (i) Let $a, b \in B$ with $a \notin b$. Then there exists $x \in D(B, \underline{2})$ with $x(a) * x(b)$. Since $\Phi$ is onto, we can find $g \in \mathbf{K}(B, \underline{P})$ such that $\Phi(g)=x$. This means that

$$
\alpha(g(a))=(\Phi(g))(a)=x(a) \nless x(b)=(\Phi(g))(b)=\alpha(g(b)) .
$$

Since $\alpha$ preserves $\preccurlyeq$, we conclude that $g(a) * g(b)$.

(ii) By interchanging the roles of $\leqslant$ and $\leqslant$ in the proof of (i) we obtain the proof of (ii).

The maps $\alpha$ and $\Phi$ play a fundamental role in Davey (1982) and in Davey and Werner (1983b), (1985). The ontoness of $\Phi$ and $\Phi^{*}$ is proved explicitly for Stone algebras in Davey (1978) and for double Stone algebras in Davey (1982). The general theory in Davey and Werner (1983b), (1985) yields indirect proofs of the ontoness of $\Phi$ for these varieties, as well as for $\mathbf{L}_{n}(2 \leqslant n<\omega)$, and for Ockham algebras, and the subvarieties $\mathbf{P}_{m, n}$.

Given $A \in \mathbf{K}$, we have two order relations $\leqslant$ and $\leqslant$ on $\mathbf{K}(A, \underline{P})$, induced respectively by the pointwise extensions of $\leqslant$ on $\underline{P}$ and of $\leqslant$ on $\underset{\sim}{P}$. It is natural to ask when these coincide, either universally for $A \in \mathbf{K}$ or for a particular algebra $A$. The containment $\leqslant \subseteq \leqslant$ on $\mathbf{K}(A, \underline{P})$ is just (H1) for $A$, and we have $\preccurlyeq \subseteq \leqslant$ on $\mathbf{K}(A, \underline{P})$ whenever $\preccurlyeq \subseteq \leqslant$ on $P$. In the next section we shall see that these conditions hold quite commonly, but not always. In general we have

LEMMA 4.14. The orders $\leqslant$ and $\preccurlyeq$ on $\mathbf{K}(A, \underline{P})$ coincide for every $A \in \mathbf{K}$ if and only if

(i) $a \nless b$ in $P$ implies that $p(a) \notin p(b)$ in $\underline{P}$ for some unary term function $p$ on $\underline{P}$,

(ii) $a \notin b$ in $\underline{P}$ implies that $q(a) * q(b)$ in $\underset{\sim}{P}$ for some unary term function $q$ on $\underline{P}$.

Proof. The first part comes from Lemma 4.9. The second is obtained by noting that a valid result is still obtained if $\leqslant$ and $\leqslant$ are interchanged in Lemma 4.9.

LEMMA 4.15. Suppose that on $P,(x \leqslant y \& x \leqslant y)$ can be expressed as a conjunct $\tau(x, y)$ of atomic formulas in two free variables, the operations in $G$, and the relations in $R$. Then $\leqslant \supseteq \preccurlyeq$ on $X=\mathbf{K}(A, \underline{P})$ if and only if

$$
X \vDash(\forall x, y \in X) \sigma(x, y) \text {, where } \sigma(x, y):=(x \preccurlyeq y \Rightarrow \tau(x, y)) .
$$

Proof. Suppose that $\leqslant \supseteq \leqslant$ on $X$. Take $x, y \in X$ with $x \leqslant y$. Then $x \leqslant y$ and $x \leqslant y$, so

$$
(\forall a \in A)(x(a) \preccurlyeq y(a) \text { and } x(a) \leqslant y(a))
$$


that is, $(\forall a \in A) \underset{\sim}{P} F \tau(x(a), y(a))$. The hypothesis on $\tau$ implies that this lifts to $X \subseteq P^{A}$, so that $X \vDash \tau(x, y)$. Hence $X \vDash(\forall x, y \in X) \sigma(x, y)$, as required.

Conversely, suppose that $X \vDash(\forall x, y \in X) \sigma(x, y)$. Let $x, y \in X$ with $x \leqslant y$. Then $X \vDash \tau(x, y)$, so that

$$
\begin{aligned}
& (\forall a \in A) \quad \underset{\sim}{P} \vDash \tau(x(a), y(a)), \\
\Rightarrow(\forall a \in A) & \underset{\sim}{P} \vDash x(a) \leqslant y(a) \text { and } x(a) \leqslant y(a) \\
\Rightarrow(\forall a \in A) & \underset{\sim}{P} \vDash x(a) \leqslant y(a),
\end{aligned}
$$

whence $x \leqslant y$, as required.

A corresponding result holds with $\leqslant$ and $\preccurlyeq$ interchanged, but we shall not need it.

\section{Examples involving Davey-Werner dualities}

This section illustrates the preceding theory. The Davey-Werner dualities for the varieties we consider are established in Davey and Werner (1983a), with the exception of those for Ockham algebras and the subvarieties $\mathbf{P}_{m, n}$, which appear in Davey and Werner (1983b), (1985). We shall be interested in particular in whether, for the variety $\mathbf{K}$ and its dual $\mathfrak{X}$, the conditions (0) and any of (II), (II)* and (III) (from Theorem 4.11) are satisfied. If they are, then $\operatorname{Cat}(\mathbf{K})=$ $E(\operatorname{Cocat}(\mathfrak{X}))$.

In all our examples, $(0)$ holds, since $\preccurlyeq$ is amongst the relations $R$ on $\underset{\sim}{P}$. To verify (III), all we need to know is the structure of $\underline{P}=(P ; F) \in \mathbf{K}$ and of $\stackrel{P}{P}=(P ; \tau, G, R) \in \mathfrak{X}$. To check (II)* we might invoke Proposition 4.13 , where it is applicable. However, even when $\Phi$ and $\Phi^{*}$ are known explicitly, more work is involved than in testing (III).

Bounded DISTRIBUTIVE LATTICES. In this case $\underline{P}$ is the 2-element chain, qua bounded distributive lattice, and $\underset{P}{P}$ is the 2-element chain, qua ordered set. Remark 4.12 shows that (II) is trivially satisfied. The Davey-Werner duality coincides with the D-P duality, for which we already known that (I) holds (by Lemma 2.2).

Stone Algebras and double Stone algebras [Davey and Werner (1983a), pages $180-183]$. The variety of Stone algebras is $\operatorname{USP}(3)$ where $\underline{3}:=(\{0, c, 1\}$; 
$\wedge, \vee, *, 0,1)$, where $0<c<1,1^{*}=c^{*}=0$, and $0^{*}=1$. The object $\underset{2}{3}$ may be taken to be $3:=(\{0, c, 1\} ; \alpha, \preccurlyeq)$, where $\leqslant$ is given by

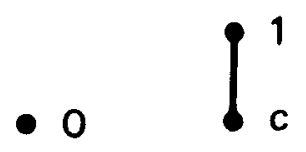

and $\alpha\left(=^{* *}\right)$ is the endomorphism defined by $\alpha(0)=0, \alpha(1)=\alpha(c)=1$.

We check (III). If $a \leqslant b$ and $a^{*} \leqslant b^{*}$ in 3 , then necessarily $a \leqslant b$ in 3 . Thus (III)(a) holds. Since $\leqslant \subseteq \leqslant$, (III)(b) is trivial.

Similar arguments can be given when $\mathbf{K}$ is the variety of double Stone algebras; again (III) holds.

OCKHAM ALGEBRAS [Davey and Werner (1983b), (1985)]. The variety $\mathbf{O}$ of Ockham algebras is $\mathbb{\Lambda S P}\left(\underline{2}^{\mathbf{N}}\right)$ with $\underline{2}^{\mathbf{N}}=\left(2^{\mathbf{N}} ; \wedge, \vee, \sim, 0,1\right)$, where the negation, $\sim$, is given by

$$
(\sim a)(n)=1 \Leftrightarrow a(n+1)=0 \quad(n \in \mathbb{N})
$$

(regarding $a \in 2^{\mathbb{N}}$ as a function $a: \mathbb{N} \rightarrow\{0,1\}$ ), and the lattice operations are defined pointwise from 2 . The dual object is

$$
2^{\mathbf{N}}=\left(2^{\mathbf{N}} ; \tau, g, \preccurlyeq\right),
$$

where $\tau$ is the usual product topology; the Ockham map $g$ is defined by

$$
g(a)(n)=1 \Leftrightarrow a(n+1)=1 \quad\left(n \in \mathbb{N}, a \in 2^{\mathbb{N}}\right) ;
$$

and $\leqslant$ is the alternating order given by $a \leqslant b$ if and only if $a(n) \leqslant b(n)$ for $n$ even, and $a(n) \geqslant b(n)$ for $n$ odd. Suppose that $a \leqslant b$ and $\sim a \leqslant \sim b$. This happens precisely if $a(0) \leqslant b(0)$ and $a(n)=b(n)$ for all $n \geqslant 1$, and under these conditions we have $a \leqslant b$. In the same way, $a \leqslant b$ and $g(a) \leqslant g(b)$ imply that $a(0) \leqslant b(0)$ and $a(n)=b(n)$ for all $n \geqslant 1$, whence $a \leqslant b$. Hence (III) holds.

The subvarieties $\mathbf{P}_{m, n}$ of $\mathbf{O}$ [Davey and Werner (1985)]. The variety $\mathbf{P}_{m, n}$ is generated by $\left(\underline{2}_{n}^{m}\right)=\left(2^{m} ; \vee, \wedge, \sim, 0,1\right)$, where $\underline{2}_{n}^{m}$ has lattice operations defined pointwise from $\underline{2}$, and $\sim$ (depending on $n$ ) is given, for $a \in \underline{2}^{m}$, by

$$
(\forall k<m)(\sim a)(k)=1 \text { if and only if } a\left(\gamma^{k+1}(0)\right)=0,
$$

where

$$
\gamma(k)= \begin{cases}k+1 & \text { if } k<m-1 \\ n & \text { if } k=m-1\end{cases}
$$

(Here $\mathbf{P}_{2,0}$ is the variety of de Morgan algebras; see Davey and Werner (1983a), page 178.) The structure $2_{n}^{m}:=\left(2^{m} ; g, \preccurlyeq\right)$, where $g: 2^{m} \rightarrow 2^{m}$ is defined by

$$
g(a)(k)=1 \quad \text { if and only if } a(\gamma(k))=1 \quad\left(a \in 2^{m}, 0 \leqslant k<m\right)
$$


and $\preccurlyeq$ is an order relation whose definition is different in the cases $(m-n)$ even and $(m-n)$ odd.

When $(m-n)$ is even, $a \leqslant b$ if and only if $a(k) \leqslant b(k)$ for $k$ even, and $a(k) \geqslant b(k)$ for $k$ odd. When $(m-n)$ is odd, we have $a \preccurlyeq b$ if and only if, for $k<n, a(k) \leqslant b(k)$ if $k$ is even, and $a(k) \geqslant b(k)$ if $k$ is odd, and $a(k)=b(k)$ for $n \leqslant k<m$. (These orders are such that the obvious mapping of $2_{\sim}^{m}$ into $2^{\mathbb{N}}$ is order-preserving.)

Suppose that $a \leqslant b$ and $\sim a \leqslant \sim b$ in $\underline{2}_{n}^{m}$. Then, for all $k$, we have $a\left(\gamma^{k}(0)\right) \leqslant$ $b\left(\gamma^{k}(0)\right)$, while $a\left(\gamma^{k+1}(0)\right) \geqslant b\left(\gamma^{k+1}(0)\right)$. Hence $a(k)=b(k)$ for $1 \leqslant k \leqslant m-1$, and $a(0) \leqslant b(0)$. Irrespective of whether $m-n$ is even or odd, we have $a \leqslant b$. Thus (III)(a) holds.

We now suppose that $a \preccurlyeq b$ and $g(a) \preccurlyeq g(b)$ in $2_{n}^{m}$. Whether $m-n$ is even or odd, these conditions yield $a(0) \leqslant b(0)$ and $a(k)=b(k)$ for $1 \leqslant k \leqslant m-1$. Hence $a \leqslant b$. Thus (III)(b) holds.

Summing up our results, we have proved

THEOREM 5.1. Suppose that $\mathbf{K}$ is any one of the varieties

(i) Bounded distributive lattices,

(ii) Stone algebras or dual Stone algebras,

(iii) Double Stone algebras,

(iv) Ockham algebras,

(v) $\mathbf{P}_{m, n}(m>n \geqslant 0)$.

Then for all $A, B \in \mathbf{K}, \mathbf{K}(A, B)$ is order-isomorphic to $\mathfrak{X}(D(B), D(A))$, where $\mathfrak{X}$ is the category dual to $\mathbf{K}=\mathbb{I S P}(\underline{P})$ for the Davey-Werner duality, and $D(A):=\mathbf{K}(A, \underline{P})$.

Each of the varieties listed in Theorem 5.1 has a piggyback duality. In fact there exists in each case a map $\alpha: P \rightarrow \underline{2}$ which is order-preserving both for $\leqslant$ and for $\leqslant$ such that $\Phi:=\alpha \circ-: \mathbf{K}(A, \underline{P}) \rightarrow \mathbf{D}(A, \underline{2})$ is a bijection. For Stone algebras and double Stone algebras the maps $\alpha, \Phi$ and $\Theta:=\Phi^{-1}$ are given in Davey (1982), and it is shown that $\Phi$ and $\Theta$ are order-preserving if $\mathbf{K}(A, \underline{P})$ carries the order $\leqslant$. It is easily seen that $\Theta$ is also order-preserving if $\mathbf{K}(A, \underline{P})$ has order $\leqslant$ (and $\Phi$ certainly is, since $\alpha: \underset{\sim}{P} \rightarrow \underline{2}$ is order-preserving). It follows that we have order-isomorphisms.

$$
(\mathbf{K}(A, \underline{P}) ; \leqslant) \underset{\boldsymbol{\Theta}}{\stackrel{\Phi}{\rightleftarrows}}(\mathbf{D}(A, \underline{2}) ; \leqslant) \underset{\Phi}{\stackrel{\Theta}{\rightleftarrows}}(\mathbf{K}(A, \underline{P}) ; \preccurlyeq) .
$$

Consequently, for every $A$, the two orders on $\mathbf{K}(A, \underline{P})$ are the same. This can also be seen from Lemma 4.14 .

Now consider $K=\mathbf{O}$, the variety of Ockham algebras. Here the map $\alpha: 2^{\mathbf{N}} \rightarrow 2$ is defined to be the projection onto the first coordinate; it is order-preserving for 
both orders. Define $\Theta$ by

$$
(\forall x \in \mathbf{D}(A, \underline{2}))(\forall a \in A) \Theta(x)(a)(n)=1 \Leftrightarrow \begin{cases}x\left(\sim^{n} a\right)=1 & (n \text { even }), \\ x\left(\sim^{n} a\right)=0 & (n \text { odd }) .\end{cases}
$$

It is routine to verify that $\Theta$ is a 2 -sided inverse for $\Phi=\alpha \circ-$, and that $\left(\mathbf{O}\left(A, \underline{2}^{\mathbf{N}}\right) ; \preccurlyeq\right)$ is order-isomorphic to $\mathbf{D}(A, \underline{2})$. Further, $\mathbf{D}(A, \underline{2})$ is order-isomorphic to $\left(\mathbf{O}\left(A, \underline{2}^{\mathbf{N}}\right) ; \leqslant\right)$ if and only if the $g$-map is contant on the order-components of $\left(\mathbf{O}\left(A, \underline{2}^{\mathrm{N}}\right) ; \preccurlyeq\right)$. This can be obtained from Lemma 4.14 (taking $\tau(x, y)$ to be $x \leqslant y \& g(x)=g(y)$ ), or by ascertaining when $\Theta$ is $\preccurlyeq$-preserving.

KLEENE ALGEBRAS. It is not true that the conditions of Theorem 4.11 are met for every variety $\mathbf{K}$ of distributive-lattice-ordered algebras which has a tractable Davey-Werner duality. We consider the variety $\mathbf{K}$ of Kleene algebras, whose duality was described in Davey and Werner (1983a), pages 175-178. The generating algebra for $\mathbf{K}$ is

$$
\underline{K}:=(\{0, c, 1\} ; \vee, \wedge, \sim, 0,1) \overbrace{\underline{K}}^{c}
$$

where $(\{0, c, 1\} ; \vee, \wedge, 0,1)$ is the chain with $0<c<1$, and $\sim$ is given by $\sim 0=1, \sim 1=0$, and $\sim c=c$. We choose

$$
\underset{\sim}{K}=\left(K=\{0, c, 1\} ; \preccurlyeq, K_{0},-\right) .
$$

Here $\preccurlyeq$ is the order

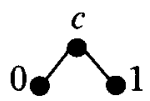

$K_{0}$ is the unary relation $\{0,1\}$ on $K$ (the minimal elements of $(K ; \preccurlyeq)$ ), and - is the binary relation $K^{2} \backslash\{(0,1),(1,0)\}$, which may be characterised as all pairs of comparable elements in $(K ; \preccurlyeq)$.

It is proved in Davey and Werner (1983a), page 175, that the dual category $\mathfrak{X}$ consists of structures $\left(X ; \tau, \preccurlyeq, X_{0},-\right)$ where $(X ; \tau, \preccurlyeq)$ is a member of $\mathbf{P}, X_{0}$ is a distinguished closed subspace (possibly empty), and - is a closed binary relation satisfying

(i) $(\forall x \in X) x-x$, and

(ii) $(\forall x, y \in X) x-y$ and $x \in X_{0}$ imply $x \leqslant y$,

(iii) $(\forall x, y, z \in X) x-y$ and $y \leqslant z$ imply $x-z$. 
Proposition 5.2. Let $\mathbf{K}$ be the variety of Kleene algebras, let $\mathfrak{X}$ be the dual category defined above, and let $\underline{K}$ and $\underset{\tilde{K}}{\mathrm{~b}}$ be as above. Then

(i) (III)(a) holds (and so (H1) holds for every $A \in \mathbf{K}$ and (H2)* holds for every $X \in \mathfrak{X})$.

(ii) (a) (H2) fails for $A=\underline{K}$; (H1)* fails for $X=F \mathfrak{X}(1)$;

(b) (H1)* fails for $X=\underset{\sim}{K}$; (H2) fails for $A=F \mathbf{K}(1)$;

(c) (III)(b) fails.

(iii) $\mathbf{K}(F \mathbf{K}(1), \underline{K})$ is not order-isomorphic to $\mathfrak{X}(D(\underline{K}), D(F \mathbf{K}(1)))$ (that is, to $\mathfrak{X}(F \mathfrak{X}(1), K)$ ).

(iv) Let $A \in \mathbf{K}$. Then $\leqslant$ and $\leqslant$ coincide on $\mathbf{K}(A, \underline{K})$ if and only if $A / \Theta$ is Boolean, where $\Theta$ is the lattice-congruence on $A$ given by $a \equiv b(\Theta) \Leftrightarrow a \vee(d$ $\wedge \sim d)=b \vee(d \wedge \sim d)$ for some $d \in A$.

[Given our earlier results, there is obvious redundancy here. However, it is instructive to see how all the various conditions work or fail in a particular case.]

Proof. (i) and (ii) are straightforward. To prove (iii) it suffices to check that $(\mathbf{K}(F \mathbf{K}(1), \underline{K}) ; \leqslant)$ is a 3 -element antichain and $(\mathfrak{X}(F \mathfrak{X}(1), \underline{K}) ; \preccurlyeq)$ is orderisomorphic to $K$.

(iv) Let $x, y \in \mathbf{K}(A, \underline{K})$. Then $x \leqslant y$ if and only if $x(a) \leqslant y(a)$ for all $a \in A$. But then $\sim x(a)=x(\sim a) \leqslant y(\sim a)=\sim y(a)$ for all $a \in A$. Since $\sim$ is order-reversing on $\underline{K}, \leqslant$ is the discrete order on $\mathbf{K}(A, \underline{K})$.

Cornish and Fowler (1979) (Theorem 2.2) prove that the Priestley dual of $A / \Theta$ is the subspace $\bar{X}=\{x \in \mathbf{D}(A, \underline{2}) \mid x \leqslant g(x)\}$ of $\mathbf{D}(A, \underline{2})$. Hence it remains to prove that $\leqslant$ on $\mathbf{K}(A, \underline{K})$ is discrete if and only if $\leqslant$ on $\bar{X}$ is discrete. For any $z \in \bar{X}$ and any $a \in A$, we have

$$
z(a)=1 \Leftrightarrow g(z)(\sim a)=0 \Rightarrow z(\sim a)=0 .
$$

Fix $z \in \bar{X}$ and define $I_{z}=\{a \in A \mid z(\sim a)=1\}=\sim^{-1}\left(z^{-1}(1)\right)$. Since $z$ is a homomorphism and $\sim$ is a dual lattice isomorphism, $I_{z}$ is a prime ideal of $A$. Moreover $I_{z} \cap \sim I_{z}=\varnothing$. Hence we may define $\bar{z} \in \mathbf{K}(A, \underline{K})$ by

$$
\bar{z}(a)= \begin{cases}0 & \text { if } a \in I_{z}, \\ c & \text { if } a \in A \backslash\left(I_{z} \cup \sim I_{z}\right), \\ 1 & \text { if } a \in \sim I_{z} .\end{cases}
$$

Suppose the order on $\bar{X}$ is not discrete. Then there exist $x, y \in \bar{X}$ with $x<y$. Then $I_{x} \subsetneq I_{y}$, since $\sim a \in I_{y} \backslash I_{x}$. It follows that $\bar{y} \preccurlyeq \bar{x}$ (with, of course, $x \neq y$ ). Thus, $\preccurlyeq$ on $\mathbf{K}(A, \underline{K})$ is non-discrete. 
Conversely, suppose there exist $\phi, \psi \in \mathbf{K}(A, \underline{K})$ with $\phi \preccurlyeq \psi, \phi \neq \psi$. Then there exists $a \in A$ such that $\phi(a)=1$ and $\psi(a)=c$. Define $\alpha: \underline{K} \rightarrow \underline{2}$ by $\alpha(0)=\alpha(c)=0$ and $\alpha(1)=1$. Then $x=\alpha \circ \phi$ and $y=\alpha \circ \psi$ belong to $\mathbf{D}(A, 2)$, and $x<y$. Finally,

$$
\begin{aligned}
(\forall a \in A) g(x)(a)=0 & \Leftrightarrow x(\sim a)=1 \quad \Leftrightarrow \quad \phi(\sim a)=1 \\
& \Leftrightarrow \sim \phi(a)=1 \\
& \Leftrightarrow \phi(a)=0 \quad \Leftrightarrow \quad x(a)=0 .
\end{aligned}
$$

Hence $x$, and similarly $y$, belongs to $\bar{X}$.

Note that not only does the natural bijection $D$ in general fail to map $\mathbf{K}(A, B)$ order-isomorphically onto $\mathfrak{X}(D(B), D(A))$, but that, as (iii) shows, there is likely to be no order-isomorphism between these sets.

It is a consequence of Proposition 5.2 and Theorem 4.11 that we cannot assume that $D(\operatorname{Cat}(\mathbf{K}))=\operatorname{Cocat}(\mathfrak{X})$. It follows from Theorem 3.15 that $D(\operatorname{Cat}(\mathbf{K}))$ consists of two objects in $\mathfrak{X}$, both containing a single point and one with an empty distinguished subspace, the other not. We shall now find $\operatorname{Cocat}(\mathfrak{X})$.

THEOREM 5.3. Let $\mathfrak{X}$ be the Davey-Werner dual category for the variety of Kleene algebras. Then $\left(X ; \tau, \preccurlyeq, X_{0},-\right) \in \operatorname{Cocat}(\mathfrak{X})$ if and only if $(X ; \tau, \preccurlyeq)$ is a topological lattice, and therefore satisfies

(i) $\left|X_{0}\right| \leqslant 1$, and

(ii) $x-y$ for all $x, y \in X$.

Proof. Our method is reminiscent of that used in Section 3. We assume that, for every $Y \in \mathfrak{X}, \mathfrak{X}(Y, X)$ is a lattice (for the pointwise order induced by $\leqslant$ on $X)$. Putting $Y=D(K)$ (so $\left.|Y|=1, Y_{0}=\varnothing\right)$ we see that $X$ is a lattice. Since $X_{0}$ is a subset of the minimal elements of $X$, we have $\left|X_{0}\right| \leqslant 1$. For every $x, y \in X$, we have $x \wedge y \leqslant x, y$ and so $x-y$ (see Davey and Werner (1983a), page 177).

We now let $\mathbf{Y}$ be the full subcategory of $\mathfrak{X}$ whose objects satisfy condition (ii) above. Construct for each $Z \in \mathbf{P}$ an object $F(Z) \in Y$ by defining $F(Z)=Z$, by letting $\tau$ and $\leqslant$ be the topology and order given on $Z$, and by defining $x-y$ for all $x, y \in F(Z)$ and $(F(Z))_{0}=\varnothing$. Then $\mathbf{P}(Z,|X|)$ is order-isomorphic to $\mathfrak{X}(F(Z), X)$, where $|-|: \mathbf{Y} \rightarrow \mathbf{P}$ is the forgetful functor. Lemma 3.1 implies that $(X ; \tau, \preccurlyeq)$ is a topological lattice. Thus the stated conditions are necessary.

Conversely suppose that $X \in \mathfrak{X}$ is a topological lattice. Since $X$ satisfies (i) and (ii), it is clear that for all $Y \in \mathfrak{X}, \mathfrak{X}(Y, X)$ is a sublattice of the lattice $\mathbf{P}(Y, X)$.

A more interesting characterisation of $\operatorname{Cocat}(\mathfrak{X})$ is obtained by reinterpreting Theorem 5.3 in terms of objects in $\mathbf{K}$. Theorem 5.4 can be established directly or 
can be deduced from results of Davey and Priestley (1984) (see the introduction to that paper).

TheOREM 5.4. Let $\mathfrak{X}$ be the category dual to $\mathbf{K}$, the variety of Kleene algebras. Then $\operatorname{Cocat}(\mathfrak{X})$ consists of those objects $D(A)$ for which $A \in \mathbf{K}$ has one of the following forms:

(A) $A$ is the linear sum of $B$ and $B^{d}$, where $B$ is catalytic in $\mathbf{D}_{0}$ (the variety of lower bounded distributive lattices);

(B) $A$ is the reduced linear sum of $B$ and $B^{d}$, where $B$ is catalytic in $\mathbf{D}$.

In each case denotes the order dual, and the negation is defined in the obvious way (by 'reflection').

[A bounded distributive lattice is of type (A) if and only if its Priestley dual is the reduced linear sum of a topological lattice and its order dual, with the obvious topology. It was proved by Balbes (1980) that $L \in \operatorname{Cat}\left(\mathbf{D}_{0}\right)$ if and only if $L \oplus 1$ Cat(D). A lattice in $D$ is of type (B) if and only if its Priestley dual is the linear sum of a topological lattice and its order dual. The catalytic Kleene algebras are those of type (A) for which $|B| \leqslant 1$ and those of type (B) for which $|B| \leqslant 2$.]

\section{Colattices in varieties of algebras}

An object $L$ in a category $\mathbf{C}$ is called a lattice in $\mathbf{C}$ if the contravariant hom-functor

$$
\mathbf{C}(-, L): \mathbf{C} \rightarrow \text { Set }
$$

factors through the category Lat of all lattices; that is, for all $A \in \mathrm{C}$ there is a lattice structure on the hom-set $\mathbf{C}(A, L)$ such that for all $\phi \in \mathrm{C}(A, B)$, the induced map from $\mathbf{C}(B, L)$ to $\mathbf{C}(A, L)$ is a lattice homomorphism. Similarly $L$ is a colattice in $\mathbf{C}$ if the covariant hom-functor

factors through Lat.

$$
\mathbf{C}(L,-): \mathbf{C} \rightarrow \text { Set }
$$

Lattices and colattices in a category with finite products and coproducts have a more concrete description. We briefly outline the ideas here and refer to Davey and Werner (1983a), pages $222-226$ or Freyd (1966) for more details. If $L \in \mathrm{C}$ and $j, m$ are morphisms from $L \times L$ to $L$ (representing join and meet), then we say that $(L ; j, m)$ satisfies the absorption law $x \wedge(x \vee y)=x$, for example, if the diagram below commutes:

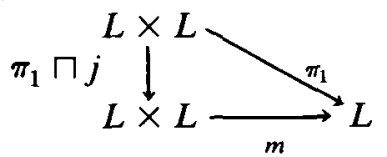


(Here $\pi_{1}$ is the first projection and, if $\phi, \psi: X \rightarrow L$ are morphisms, then $\phi \sqcap \psi: X \rightarrow L \times L$ is the obvious "product" morphism.) If $(L ; j, m)$ satisfies all the lattice axioms expressed in this way, we say that $(L ; j, m)$ is a concrete lattice in $\mathbf{C}$. By reversing all arrows and replacing products by coproducts (so that $L$ is now equipped with co-operations $j, m: L \rightarrow L * L$ ), we obtain the somewhatless-familiar concept of a concrete colattice in $\mathrm{C}$.

The following fundamental observation is standard.

LEMMA 6.1. (i) If $\mathbf{C}$ has finite products, then $L$ is a lattice in $\mathbf{C}$ if and only if there are morphisms $j, m: L \times L \rightarrow L$ such that $(L ; j, m)$ is a concrete lattice in C.

(ii) If $\mathbf{C}$ has finite coproducts, then $L$ is a colattice in $\mathbf{C}$ if and only if there are morphisms $j, m: L \rightarrow L * L$ such that $(L ; j, m)$ is a concrete colattice in $\mathbf{C}$.

Clearly if we have a full duality between $\mathbf{C}$ and $\mathfrak{X}$, then $L$ is a colattice in $\mathbf{C}$ precisely when its dual is a lattice in $\mathfrak{X}$. If $\mathfrak{X}$ is a category of structured sets, and if products in $\mathfrak{X}$ are cartesian, then the duality is a very useful tool for describing the colattices in $\mathbf{C}$, since the lattices in the category $\mathfrak{X}$ will be lattices in the usual set-theoretic sense. But if products in $\mathfrak{X}$ are not cartesian, then a lattice in $\mathfrak{X}$ may be a totally unfamiliar creature. Hence the dualities of the type introduced in Section 5 are particularly useful, since the compatibility conditions on $\underline{P}$ and $\underset{\sim}{P}$ guarantee that products in the dual category $\mathfrak{X}=\mathbb{E P}(\underset{\sim}{P})$ are cartesian. For example, Kleene algebras have a dual category of this type (which therefore has well-behaved products); but if we restrict the D-P duality to the subcategory of Kleene algebras, then products in the dual category so obtained are no longer cartesian.

A lattice-catalytic algebra $L$ in a variety $\mathbf{K}$ of lattice-ordered algebras need not be a colattice in $\mathbf{K}$ : although the map from $\mathbf{K}(L, A)$ to $\mathbf{K}(L, B)$ induced by a homomorphism $\phi \in \mathbf{K}(A, B)$ will be order-preserving, it need not be a lattice homomorphism. Consequently the following result is rather surprising.

THEOREM 6.2. Suppose that $\mathbf{K}$ is

(i) the variety $\mathbf{D}$ of bounded distributive lattices,

(ii) the variety $\mathbf{B}_{n}$ of distributive p-algebras $(0 \leqslant n \leqslant \omega)$,

(iii) the variety $\mathbf{B}_{n}^{m}$ of distributive double p-algebras $(1 \leqslant m, n \leqslant \omega)$,

(iv) the variety $\mathbf{O}$ of Ockham algebras,

(v) the subvariety $P_{m, n}$ of Ockham algebras $(m>n \geqslant 0)$. Then every catalytic algebra in $\mathbf{K}$ is a colattice in $\mathbf{K}$. 
Proof. Let $\mathfrak{X}=D(\mathbf{K})$ be the restricted $\mathbf{D}-\mathbf{P}$ dual of $\mathbf{K}$. By the results of Section 3 , if $X \in \mathfrak{X}$ is cocatlytic in $\mathfrak{X}$, then $X$ is lattice ordered. If $\mathbf{K}$ is $\mathbf{D}, \mathbf{B}_{0}, \mathbf{B}_{1}$, $\mathbf{B}_{\mathbf{l}}^{1}, \mathbf{O}$ or $\mathbf{P}_{m, n}$, then products in $\mathfrak{X}$ are cartesian, while if $\mathbf{K}$ is any $\mathbf{B}_{n}$ or $\mathbf{B}_{n}^{m}$, then the product $X \times X$ in $\mathfrak{X}$ is cartesian at least when $X$ is cocatalytic in $\mathfrak{X}$. Consequently, in each case, if $X$ is cocatalytic in $\mathfrak{X}$, the lattice order on $X$ will make it into a lattice in $\mathfrak{X}$ precisely when the induced join and meet operations are $\mathfrak{X}$-morphisms from $X \times X$ to $X$. With this observation the result follows easily from the characterisations of the cocatalytics given in Section 3.

It is important to realise that, when we claim that $A \in \mathbf{K}$ is a colattice in $\mathbf{K}$, we are only saying that there exists a colattice structure on $A$; this structure need not be unique. For example, if $A \in \mathbf{D}$ has dual $(X ; \tau, \preccurlyeq)$ in $\mathbf{P}$ and there is some lattice structure $(X ; \vee, \wedge)$ such that $\vee$ and $\wedge$ are continuous and $\preccurlyeq$-preserving, then $(X, \vee, \wedge)$ is a lattice in $\mathbf{P}$ and so induces a colattice structure on $A$. In particular, if $X$ is cocatalytic in $\mathbf{P}$ with resulting lattice order $(X ; \vee, \wedge)$, then both $(X ; \vee, \wedge)$ and $(X ; \wedge, \vee)$ make $X$ into a lattice in $\mathbf{P}$ and so induce distinct colattice structures on $A$ whenever $|A|>2$. Examples of lattices in $\mathbf{P}$ and of members of $\mathbf{P}$ which can never be lattices in $\mathbf{P}$ are easily found: in Examples 1 and 2 below the lattice on the right makes the ordered set on the left into a lattice in $\mathbf{P}$, while Examples 3 and 4 cannot be made into lattices in $\mathbf{P}$. (A fuller discussion of the structure of lattices in $\mathbf{P}$ will appear elsewhere.)

1.
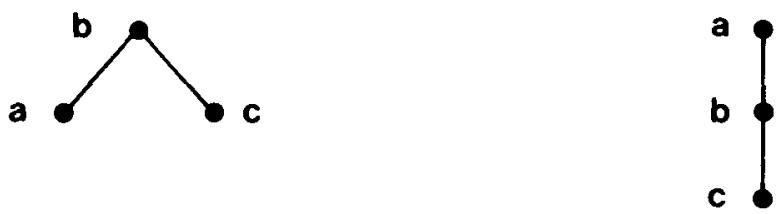

2.
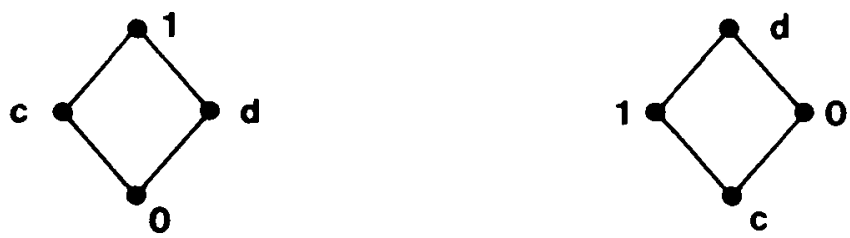

3.

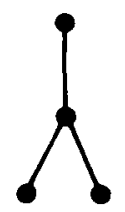

4.

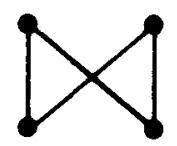

As was remarked earlier, whenever products in the dual category $\mathfrak{X}$ are cartesian, every lattice $X$ in $\mathfrak{X}$ is a lattice in the usual set-theoretic sense. If the 
original order on $X$ is a lattice order with respect to which $X$ becomes a lattice in $\mathfrak{X}$, we shall say that $X$ is an order compatible lattice in $\mathfrak{X}$.

Theorem 6.3. Let $\mathbf{K}$ be $\mathbf{D}, \mathbf{B}_{0}, \mathbf{B}_{1}, \mathbf{B}_{1}^{1}, \mathbf{O}$ or $\mathbf{P}_{n}^{m}$ (for $m>n \geqslant 0$ ). Then $A$ is catalytic in $\mathbf{K}$ if and only if the dual of $A$ is an order-compatible lattice in the Davey-Werner dual category $\mathfrak{X}$ or equivalently, in the restricted $\mathbf{D}-\mathbf{P}$ dual $\mathbf{D}(\mathbf{K})$.

Proof. Firstly note that the statement of the result makes sense since products in the Davey-Werner dual categories are cartesian, and for the varieties listed the Davey-Werner dual category and the restricted D-P dual category are isomorphic. By the previous result it remains to see that the cocatalytic objects in $\mathfrak{X}$ are the only order-compatible lattices in $\mathfrak{X}$. Hence we must show that if the order on $X$ is a lattice order, and if join and meet are $\mathfrak{X}$-morphisms from $X^{2}$ to $X$, then $X$ is cocatalytic in $\mathfrak{X}$.

From the results of Section 3, this is trivial in the cases where $\mathbf{K}$ is $\mathbf{D}, \mathbf{B}_{0}, \mathbf{B}_{1}$, or $\mathbf{B}_{1}^{1}$. If $\mathbf{K}$ is $\mathbf{O}$ or $\mathbf{P}_{m, n}$, then, since $\vee$ on $X \in \mathfrak{X}$ preserves the $g$-map, we have

$$
\begin{aligned}
x & \leqslant 1 \quad \Rightarrow \quad g(x) \geqslant g(1) \\
& \Rightarrow \quad g(x)=g(x) \vee g(1)=g(x \vee 1)=g(1) .
\end{aligned}
$$

Hence $g$ is constant on $X$, and consequently $X$ is cocatalytic in $\mathfrak{X}$.

For Kleene algebras the concept of an order-compatible lattice in the dual category makes sense only in the Davey-Werner dual.

THEOREM 6.4. Let $\mathbf{K}$ be the variety of Kleene algebras and let $\mathfrak{X}$ be its Davey-Werner dual category. Then the following are equivalent for $A \in \mathbf{K}$ :

(i) $A$ is a colattice in $\mathbf{K}$ whose dual is an order-compatible lattice in $\mathfrak{X}$;

(ii) the dual of $A$ is a topological lattice;

(iii) the dual of $A$ is cocatalytic in $\mathfrak{X}$;

(iv) $A$ is a linear sum or reduced linear sum of the form described in Theorem 5.4.

Proof. The equivalence of (ii), (iii), and (iv) is the content of Theorems 5.3 and 5.4, and (i) $\Rightarrow$ (ii) is trivial. If $X \in \mathfrak{X}$ is a topological lattice, then $\left|X_{0}\right| \leqslant 1$ and $x-y$ for all $x, y \in X$ (see Theorem 5.3). It follows at once that join and meet are $\mathfrak{X}$-morphisms from $X \times X$ to $X$, and hence that $X$ is an order-compatible lattice in $\mathfrak{X}$.

There are several circumstances under which colattices and, more generally, coalgebras occur naturally. The free algebra on one generator in $\mathbf{K}$ carries a natural K-coalgebra structure (see Davey and Werner (1983a), pages 225-226 or 
Freyd (1966)). So if $\mathbf{K}$ is a variety of lattice-ordered algebras, $F \mathbf{K}(1)$ will carry a natural colattice structure. The compatibility conditions on $\underline{P}$ and $P$ in Section 5 say precisely that $\underline{P}$ is a K-algebra structure on the object $\underset{\sim}{P}$ in $\mathfrak{X}=\mathbb{E P}(\underset{\sim}{P})$. If $\mathbf{K}=\operatorname{ISP}(\underline{P})$ is a class of lattice-ordered algebras, then the lattice structure on $P$ inherited from $\underline{P}$ is the dual of the natural colattice structure on $F \mathbf{K}(1)$ referred to above. (Note that $g \mapsto g\left(x_{1}\right)$ is an $\mathfrak{X}$-morphism from $D(F \mathbf{K}(1))=\mathbf{K}(F \mathbf{K}(1), \underline{P})$ to $P$ : see Davey and Werner (1983a) for details.) The covariant version of Freyd's Representability Theorem (see Freyd (1966)) tells us that if $\mathbf{K}$ and $\mathbf{L}$ are varieties and $G: \mathbf{K} \rightarrow \mathbf{L}$ is a functor which has a left adjoint, then there exists an $\mathbf{L}$-coalgebra $L$ in $\mathbf{K}$ such that $G$ is naturally isomorphic to the hom-functor $\mathbf{K}(L,-): \mathbf{L} \rightarrow \mathbf{K}$. If $\mathbf{L}$ is a variety of lattices or lattice-ordered algebras, then $L$ will be a colattice in $\mathbf{K}$.

\section{References}

R. Balbes (1980), 'Catalytic distributive Lattices', Algebra Universalis 11, 334-340.

R. Balbes and Ph. Dwinger (1974), Distributive lattices (University of Missouri Press, Columbia, Missouri).

W. H. Cornish and P. R. Fowler (1979), 'Coproducts of Kleene alglebras', J. Austral. Math. Soc. (Series A) 27, 209-220.

B. A. Davey (1978), 'Toplogical duality for prevarieties of universal algebras', Advances in Math., Suppl. Studies 1, 61-99.

B. A. Davey (1982), 'Dualities for Stone algebras, double Stone algebras, and relative Stone algebras', Colloq. Math. 46, 1-14.

B. A. Davey and D. Duffus (1982), 'Exponentiation and duality' (in Ordered sets, edited by I. Rival, NATO Advanced Study Institutes Series, D. Riedel Publishing Company, Dordrecht, pp. 43-95).

B. A. Davey and H. A. Priestley (1984), 'Generalized piggyback dualities and applications to Ockham algebras, Houston J. Math., to appear.

B. A. Davey and H. Werner (1983a), 'Dualities and equivalences for varieties of algebras', Colloq. Math. Soc. János Bolyai 33, 101-275.

B. A. Davey and H. Werner (1983b), 'Piggyback dualities', Colloq. Math. Soc. János Bolyai, to appear.

B. A. Davey and H. Werner (1985), 'Piggyback-Dualitäten', Bull. A ustral. Math. Soc. 32, 1-32.

P. Freyd (1966), 'Algebra valued functors in general and tensor products in particular', Colloq. Math. 14, 89-106.

G. Gierz, K. H. Hofmann, K. Keimel, J. D. Lawson, M. Mislove and D. S. Scott (1980), $A$ compendium of continuous lattices, (Springer-Verlag, Berlin, Heidelberg, New York).

M. S. Goldberg (1981), 'Distributive Ockham algebras: free algebras and injectivity', Bull. Austral. Math. Soc. 24, 161-203.

T. G. Kucera and B. Sands (1978), 'Lattices of lattice homomorphims', Algebra Universalis 8, 180-190.

H. A. Priestley (1982a), 'Catalytic distributive lattices and compact zero-dimensional topological lattices', Algebra Universalis, to appear. 
H. A. Priestley (1982b), 'Algebraic lattices as duals of distributive lattices' (in Proceedings of the conference on topological and categorical aspects of continuous lattices, Bremen, 1982, Lecture Notes in Pure and Applied Mathematics, Marcel Dekker, New York), to appear.

H. A. Priestley (1982c), 'Ordered sets and duality for distributive lattices' (in Proceedings of the conference on ordered sets and their applications, Lyon, 1982, Annals of Discrete Mathematics, North-Holland, Amsterdam, London), to appear.

A. Urquhart (1979), 'Lattices with a dual homomorphic operation', Studia Logica 38, 201-209.

Department of Pure Mathematics

La Trobe University

Bundoora

Victoria 3083

Australia
Mathematical Institute

$24 / 29$ St. Giles

Oxford OX1 3LB

England 